Gangolells M., Casals M., Forcada N., Macarulla M. Predicting on-site environmental impacts of municipal engineering works. Environmental Impact Assessment Review, 2014, 44: 43-57. < 10.1016/j.eiar.2013.08.004>.

Final version available at:

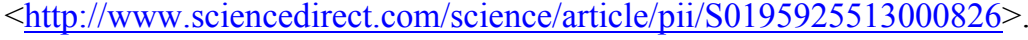

\title{
Predicting on-site environmental impacts of municipal engineering works
}

\author{
Marta Gangolells ${ }^{\mathrm{a}^{*}}$, Miquel Casals ${ }^{\mathrm{b}}$, Núria Forcada ${ }^{\mathrm{c}}$, Marcel Macarulla ${ }^{\mathrm{d}}$ \\ ${ }^{a}$ Corresponding author: Universitat Politècnica de Catalunya, Department of Construction Engineering, \\ Group of Construction Research and Innovation (GRIC), C/ Colom, 11, Ed. TR5, 08222 Terrassa \\ (Barcelona), Spain. E-mail: marta.gangolells@upc.edu. Tel: (+34) 93 7398947, Fax: (+34) 937398670.
}

${ }^{\mathrm{b}}$ Universitat Politècnica de Catalunya, Department of Construction Engineering, Group of Construction Research and Innovation (GRIC), C/ Colom, 11, Ed. TR5, 08222 Terrassa (Barcelona), Spain. E-mail: miquel.casals@upc.edu.Tel: (+34) 93 7398655, Fax: (+34) 937398670.

${ }^{c}$ Universitat Politècnica de Catalunya, Department of Construction Engineering, Group of Construction Research and Innovation (GRIC), C/ Colom, 11, Ed. TR5, 08222 Terrassa (Barcelona), Spain. E-mail: nuria.forcada@upc.edu.Tel: (+34) 93 7398153, Fax: (+34) 937398670.

${ }^{d}$ Universitat Politècnica de Catalunya, Department of Construction Engineering, Group of Construction Research and Innovation (GRIC), C/ Colom, 11, Ed. TR5, 08222 Terrassa (Barcelona), Spain. E-mail: marcel.macarulla@upc.edu. Tel: (+34) 93 7398947, Fax: (+34) 937398670.

\section{ABSTRACT}

The research findings fill a gap in the body of knowledge by presenting an effective way to evaluate the significance of on-site environmental impacts of municipal engineering works prior to the construction stage. First, 42 on-site environmental impacts of municipal engineering works were identified by means of a process-oriented approach. Then, 46 indicators and their corresponding significance limits were determined on the basis of a statistical analysis of 25 new-build and remodelling municipal engineering projects. In order to ensure the objectivity of the assessment process, direct and indirect indicators were always based on quantitative data from the municipal engineering project documents. Finally, two case studies were analysed and found to illustrate the practical use of the proposed model. The model highlights the significant environmental impacts of a particular municipal engineering project prior to the construction stage. Consequently, preventive actions can be planned and implemented during on-site activities. The results of the model also allow a comparison of proposed municipal engineering projects and alternatives with respect to the overall on-site environmental impact and the absolute importance of a particular environmental aspect. These findings are useful within the framework of the environmental impact assessment process, as they help to improve the identification and evaluation of on-site environmental aspects of municipal engineering works. The findings may also be of use to construction companies that are willing to implement an environmental management 
system or simply wish to improve on-site environmental performance in municipal engineering projects.

Keywords:

municipal engineering projects, on-site environmental impacts, construction process, EMAS, EIA

\section{INTRODUCTION}

According to the United Nations Human Settlements Programme (2009), the major urban challenges of the twenty-first century include the rapid growth of small- and medium-sized towns. Between 2007 and 2025, the annual urban population increase in developing regions is expected to be 53 million (or 2.27\%) (United Nations Human Settlements Programme, 2009). The actual rate could be even greater, as in China alone the number of people who move annually from rural to urban areas has been estimated at approximately 21.1 million (He et al., 2013). In addition, the annual urban population increase in developed regions is expected to be 3 million (or $0.49 \%$ ) between 2007 and 2025 (United Nations Human Settlements Programme, 2009). This will obviously require a great amount of municipal engineering works. Moreover, cities in developed countries have to cope with an increasingly ageing infrastructure. This problem is compounded by the fact that post-industrial European cities are characterized by dispersed urbanization (Riera and Rey, 2013). Since municipal engineering projects are concerned with public infrastructure and services provided by local government, they play a key role in improving the community's health and quality of life. Municipal engineering projects include the design, planning, construction and maintenance of streets, pavements, bicycle paths, public parks and related urban public facilities (street lighting, as well as street furniture and fixtures such as benches, bus shelters, litter bins, traffic control devices, playground equipment and road signs). The term "municipal engineering projects" also covers sanitary and storm sewer systems and municipal solid waste management and disposal facilities. Civil infrastructure (conduits and access chambers) related to utility services (water supply, electrical distribution and telecommunications networks) are also included within this term.

Although municipal engineering works have made significant contributions to sustainable development in the past, particularly in terms of social aspects, their on-site environmental impacts have often been overlooked. In most cases, municipal engineering projects are not subject to environmental impact assessment (EIA); a process by which the environmental effects of a proposed project during the construction, operation and dismantling phases are assessed at an early stage. Industrial estate development projects and urban development projects are listed in Annex II of the EIA directive (European Union, 2011), and thus they are subject to a screening process. In this case, Member States determine the need for an EIA on a case-by-case basis or according to pre-defined thresholds or criteria (size, location, etc.). Although the scope of these categories is interpreted differently by the Member States, an industrial estate development project should be understood as a specific area of land that is zoned or developed for industrial or joint industrial and business purposes, and where the necessary infrastructure is provided (European Commission, 2008). According to 
the European Commission (2008), construction projects such as housing developments, car parks, shopping centres, hospitals, universities, sports stadiums and theatres should be placed in the category of urban development projects. Projects to which the terms 'urban' and 'infrastructure' can be applied, such as the construction of sewerage and water supply networks, should also be included in this category (European Commission, 2008). Although the identification and evaluation of environmental impacts is considered integral to the EIA process, several researchers consider that this task is particularly problematic (Badr et al. 2011; Canelas et al. (2005); Peterson (2010); Androulidakis and Karakassis (2006); Kruopienè et al., 2009; Nadeem and Hameed, 2008; Toro et al. 2012; Tennøy et al., 2006). Badr et al. (2011) reviewed 45 environmental impact statements produced in Egypt and concluded that the identification and evaluation of key impacts was the area with the worst performance. One of the main weaknesses was in the prediction of impact magnitude: $69.0 \%$ of environmental impact statements within the sample were assessed as poor and only 15.5\% were considered good (Badr et al., 2011). Canelas et al. (2005) reviewed 23 environmental impact statements produced in Spain and concluded that $28 \%$ did not describe the significant environmental effects of the project in a satisfactory way. Peterson (2010) also demonstrated major deficiencies in this area. A quality assessment of 50 environmental impact studies in Estonia concluded that $55.2 \%$ of them poorly described the likely significant effects of the project (Peterson, 2010). In Greece, Androulidakis and Karakassis (2006) reviewed a sample of 37 environmental impact studies and concluded that $40 \%$ of the studies did not include impact identification and $75 \%$ of them did not conduct impact assessment. Common deficiencies were lack of transparency and inadequate explanation of the models and methods used (Tennøy et al., 2006; Badr et al., 2011), use of descriptive text instead of models (Androulidakis and Karakassis, 2006), poor quality of the methodologies used to forecast the environmental effects (Kruopiene et al., 2009), failure to quantify impact characteristics (Badr et al,. 2011), subjectivity in forecasting environmental effects (Kruopienè et al., 2009; Toro et al., 2012) and heavy reliance on qualitative analysis of impact significance (Nadeem and Hameed, 2008). According to Gómez-Navarro et al. (2009), EIAs of long life-cycle projects generally focus on the exploitation phase. Thus, on-site environmental impacts related to municipal engineering works are often overlooked. Persistent calls to conduct more detailed EIAs are based on the assumption that if an EIA is carried out more comprehensively and rigorously, it will fulfil its aims more successfully (Jay et al., 2007).

Although the EIA screening process varies significantly among countries (Martínez Orozco, 2006), most municipal engineering projects are not subject to the EIA process because of their size, location (in sensitive ecological areas in particular) or potential impact (surface area affected and duration). In these cases, environmental considerations are very limited in permit procedures. As a result, these types of projects can be executed without having assessed in advance the potential on-site environmental impacts. This is particularly true when the construction company performing the on-site works does not have sufficient environmental awareness. In other cases, municipal engineering works for industrial estate development projects or urban development projects are subject to EIA, but the literature recognizes that there are major methodological shortcomings during the early identification and evaluation of on-site environmental impacts. 
Previous research has demonstrated both the presence and importance of on-site environmental impacts (Šelih, 2007; Shen et al., 2007; Gangolells et al., 2009, 2011; Fuertes et al., 2013). Gangolells et al. $(2009,2011)$ developed a model for the early identification and assessment of on-site environmental impacts related to residential construction projects. Taking into account that the construction sector is an industry which, through its size and diversity, is able to transform the land and improve the community's health and quality of life across a wide range of projects, it becomes necessary to extend the perspective from residential building units to urbanization projects. However, to the authors' knowledge, no studies have proposed a systematic way of identifying and evaluating the significance of environmental impacts of on-site municipal engineering works. The aim of this paper is to develop a model to objectively assess the significance of on-site environmental impacts of both new-build and remodelling municipal engineering projects in advance. Following this introduction, the second section describes the methodology adopted in this research. Two case studies are reported in the third section, to illustrate a practical application of the model and to demonstrate how significant on-site environmental impacts of municipal engineering projects can be highlighted in advance. The forth section discusses the results obtained in the case studies and the final section reports the conclusions of this research and outlines future research issues.

\section{METHODOLOGY}

The methodology used in this research is adapted from Gangolells et al. $(2009 ; 2011)$ and includes the following steps:

1.Identification of on-site environmental aspects of municipal engineering projects.

2. Assessment of on-site environmental aspects of municipal engineering projects.

a. Development of indicators.

b. Determination of the significance limits.

3. Determination of the overall environmental impact of a municipal engineering project.

\subsection{Identification of on-site environmental aspects of municipal engineering projects}

The first step of this methodology was to identify on-site environmental aspects of municipal engineering works. To do this, an exhaustive preliminary analysis was carried out, using a process-oriented approach (Zobel and Burman, 2004). First, the main processes related to municipal engineering works were identified and divided into smaller process steps. The municipal engineering processes that were initially considered were (1) demolition, earthwork and waste management, (2) foundations and retaining walls, (3) closures and partition walls, (4) impermeable membranes and insulations, (5) coatings, (6) paving, (7) protection and signposting, (8) drainage and channels, (9) pipes and accessories for gases and fluids, (10) street furniture and (11) gardening. A total of 74 stages and activities related to municipal engineering works were considered in this initial environmental review. 
Second, the Eco-Management and Audit Scheme (EMAS) (European Union, 2011) was used to identify general environmental aspects. The aspects that were considered in this initial review were (1) emissions to air, (2) discharges to water, (3) avoidance, recycling, reuse, transportation and disposal of solid and other waste, particularly hazardous waste, (4) use and contamination of land, (5) use of natural resources and raw materials (including energy), (6) local issues (noise, vibration, odour, dust, visual appearance, etc.), (7) transport issues, (8) risks of environmental accidents and impacts arising or likely to arise from incidents, accidents and potential emergency situations and (9) effects on biodiversity. To increase the level of precision, some of these environmental aspects were subdivided (Lundeberg et al., 2007). For example, the consumption of water, electricity, fuel and raw materials were considered, rather than just the use of natural resources and raw materials (including energy). Finally, a total of 23 generic environmental aspects were used in this initial review.

Third, the significance rating of an environmental aspect in a particular process was determined. ISO 14004:2004 (2004) states that when criteria for significance have been established, an organization should consider environmental criteria (such as the scale, severity and duration of the impact or the type, size and frequency of an environmental aspect), applicable legal requirements (such as emission and discharge limits in permits or regulations and the concerns of internal and external interested parties (such as those related to organizational values and public image). Gangolells et al. (2009) concluded that some of these components of significance did not depend on the construction project, and thus they could be used to determine significant environmental aspects of the construction process. Therefore, the scale of the impact, its probability of occurrence and its duration are used in this early stage to determine significant environmental aspects that are common to every process in on-site municipal engineering works. According to the methodology proposed by Gangolells et al. (2009) and to reduce subjectivity during the identification of environmental aspects, a four-interval numerical scale was developed for each of the three aforementioned components of significance. The geographic scale of an environmental impact was based on a series of units. A score of " 0 " indicated no impact, " 1 " represented a geographic scale limited to the site and surrounding area, "2" had a broader scope and included local and regional areas, and " 3 " indicated that the impact had a national or international dimension (outside of the region). The probability of occurrence was defined as a progression through the various levels of likelihood. Scores "0", "1", "2" and "3" were assigned to improbable, not very likely, likely and very likely impacts respectively. The impact duration is described quantitatively in relation to the duration of the construction phase. Scores "0", " 1 ", " 2 " and " 3 " were assigned to no impact duration, less than the work phase, equal to the work phase and more than the work phase, respectively. The scale of the impact, its duration and its probability of occurrence can be cross-referenced. For example, generation of dust during earthworks is site-specific, short-term and has a high probability of occurrence, whereas generation of greenhouse gas emissions that contribute to climate change during the execution of closures and partition walls has an international scale and is persistent, but has low probability of occurrence if we exclude the transportation of materials. 
Thus, and according to Gangolells et al. (2009), the overall significance rating of an environmental impact in a particular municipal engineering process was obtained using the following expression:

$$
S G_{i}=D_{i} \cdot S_{i} \cdot P_{i}
$$

where $\mathrm{SG}_{\mathrm{i}}$ denotes the overall significance rating of an environmental impact in a specific municipal engineering process $i, D_{i}$ represents the impact duration, $S_{i}$ corresponds to the impact scale and $\mathrm{P}_{\mathrm{i}}$ denotes the probability of occurrence of the impact.

A panel of experts evaluated the significance rating of the 23 general environmental impacts in each of the 74 stages and activities initially considered. Panel members included 8 construction industry professionals and 2 academics with proven expertise in environmental management in construction. The panel of experts was provided with a matrix in which the columns were the general environmental aspects and the rows were the construction stages. For each of the intersection cells, the experts were asked to assess impact duration, impact scale and probability of occurrence. For the sake of prioritization, an environmental impact was considered significant for a specific process if its overall significance rating was greater than 3 . This level of acceptance was defined by considering an intermediate probability, duration and scale. The resulting matrix allowed us to distinguish potential environmental impacts for each municipal engineering process. As a final step, and in order to make future assessments controllable and effective, environmental aspects were aggregated with the help of the experts. As a result of this process, 42 significant environmental aspects of on-site municipal engineering works were obtained in 9 different categories: atmospheric emissions, water alteration, waste generation, soil alteration, resource consumption, local issues, transport issues, environmental accidents and effects on biodiversity (Table $1)$. 


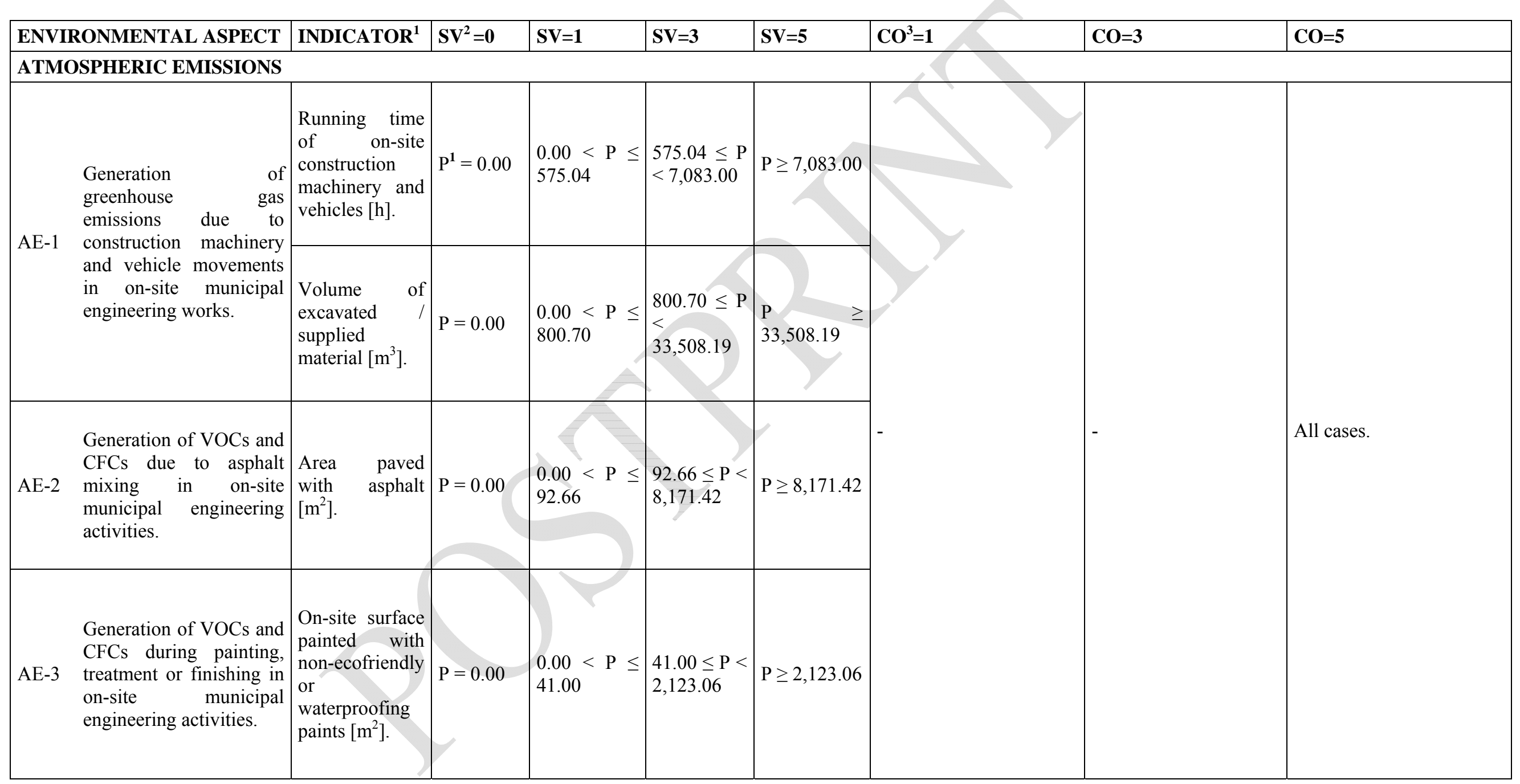




\begin{tabular}{|c|c|c|c|c|c|c|c|c|c|}
\hline \multicolumn{2}{|c|}{ ENVIRONMENTAL ASPECT } & INDICATOR $^{1}$ & $\mathrm{SV}^{2}=\mathbf{0}$ & $\mathrm{SV}=1$ & $\mathrm{SV}=3$ & SV=5 & $\mathrm{CO}^{3}=1$ & $\mathrm{CO}=3$ & $\mathrm{CO}=5$ \\
\hline \multicolumn{10}{|c|}{ WATER ALTERATION } \\
\hline WA-1 & $\begin{array}{lr}\text { Dumping of } & \text { sanitary } \\
\text { water resulting from on- } \\
\text { site } & \text { sanitary } \\
\text { conveniences } & \text { in } \\
\text { municipal engineering } \\
\text { works. }\end{array}$ & \begin{tabular}{|l} 
Average \\
number $r$ \\
workers per \\
day [number of \\
workers].
\end{tabular} & $\mathrm{P}=0.00$ & $\begin{array}{l}0.00<\mathrm{P} \leq \\
6.37\end{array}$ & $\begin{array}{l}6.37 \leq \mathrm{P}< \\
16.54\end{array}$ & $P \geq 16.54$ & $\begin{array}{l}\text { Connection to sewage } \\
\text { system. }\end{array}$ & $\begin{array}{l}\text { Dumping in septic tank } \\
\text { and/or existence of } \\
\text { previous treatment. }\end{array}$ & $\begin{array}{l}\text { Direct dumping to the } \\
\text { natural or urban } \\
\text { environment. }\end{array}$ \\
\hline WA-2 & $\begin{array}{l}\text { Dumping of water } \\
\text { resulting from the } \\
\text { execution of retaining } \\
\text { walls in on-site } \\
\text { municipal engineering } \\
\text { works. }\end{array}$ & $\begin{array}{ll}\text { Use } & \text { of } \\
\text { thixotropic } & \\
\text { fluid. } & \end{array}$ & $\begin{array}{l}\text { No use of } \\
\text { thixotropi } \\
\text { c fluid. }\end{array}$ & - & $\begin{array}{l}\text { Use of } \\
\text { thixotropic } \\
\text { fluid. }\end{array}$ & & \multirow{3}{*}{$\begin{array}{l}\text { Existence of an in situ } \\
\text { waterproof settling basin } \\
\text { or watertight tank. }\end{array}$} & \multirow{3}{*}{$\begin{array}{l}\text { Connection to sewage } \\
\text { system, dumping in } \\
\text { septic tank and/or } \\
\text { existence of previous } \\
\text { treatment. }\end{array}$} & \multirow{3}{*}{$\begin{array}{l}\text { Direct dumping to the } \\
\text { natural or urban } \\
\text { environment. }\end{array}$} \\
\hline WA-3 & $\begin{array}{l}\text { Dumping of water from } \\
\text { cleaning painting tools in } \\
\text { on-siter municipal } \\
\text { engineering works. }\end{array}$ & $\begin{array}{l}\text { On-site surface } \\
\text { painted with } \\
\text { non-ecofriendly } \\
\text { paints }\left[\mathrm{m}^{2}\right]\end{array}$ & $\mathrm{P}=0.00$ & $\begin{array}{l}0.00< \\
33.57\end{array}$ & $\begin{array}{l}33.57 \leq \mathrm{P}< \\
1,167.83\end{array}$ & $P \geq 1,167.83$ & & & \\
\hline WA-4 & $\begin{array}{l}\text { Dumping of water from } \\
\text { cleaning concrete chutes } \\
\text { or dumping of other } \\
\text { basic fluids in on-site } \\
\text { municipal engineering } \\
\text { works. }\end{array}$ & $\begin{array}{l}\text { Volume of in- } \\
\text { situ concrete } \\
{\left[\mathrm{m}^{3}\right] .}\end{array}$ & $\mathrm{P}=0.00$ & $\begin{array}{l}0.00<\mathrm{P} \leq \\
252.24\end{array}$ & $\begin{array}{l}252.24 \leq \mathrm{P} \\
<3,835.39\end{array}$ & $\mathrm{P} \geq 3,835.39$ & & & \\
\hline
\end{tabular}




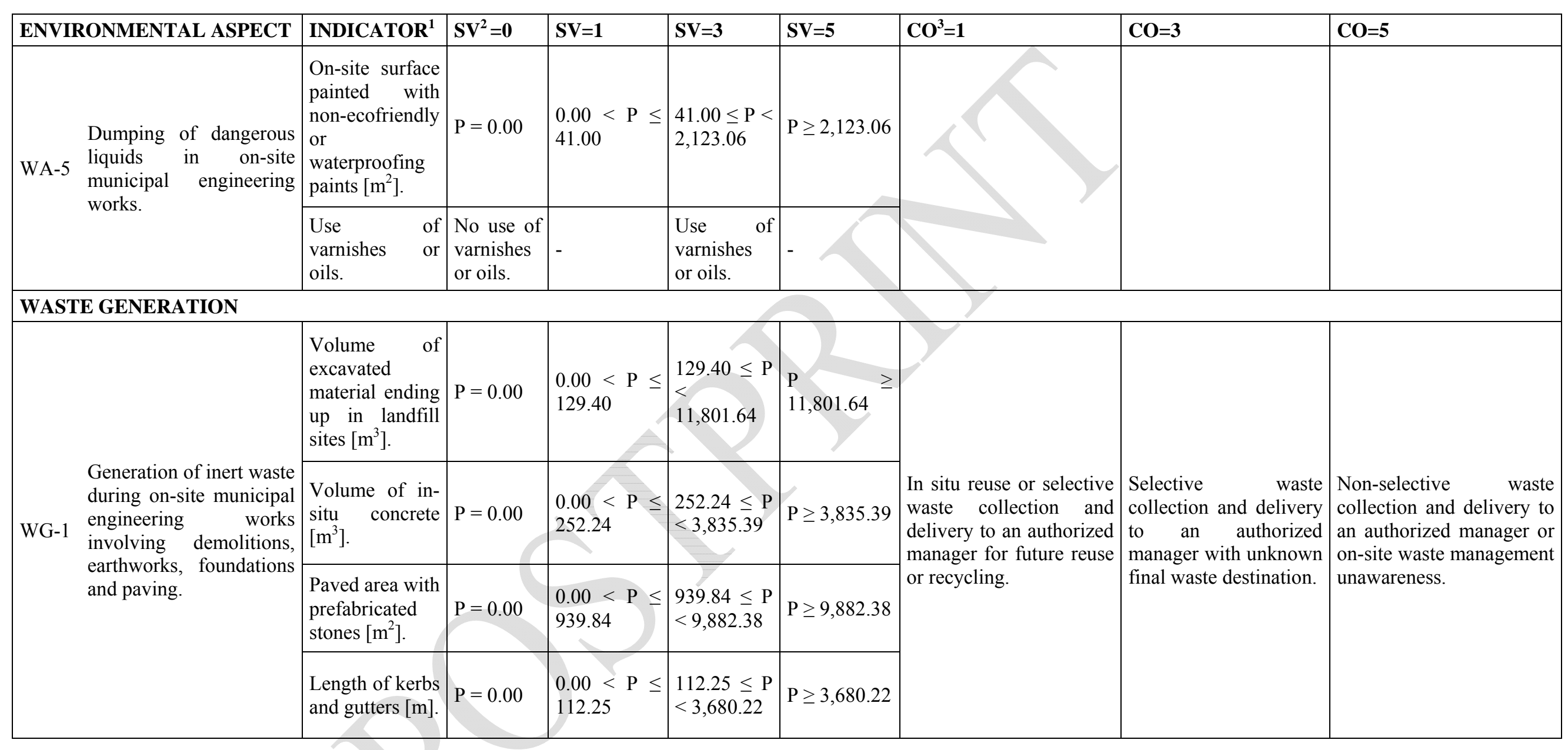




\begin{tabular}{|c|c|c|c|c|c|c|c|c|c|}
\hline ENVI & RONMENTAL ASPECT & INDICATOR $^{1}$ & $\mathrm{SV}^{2}=\mathbf{0}$ & $\mathrm{SV}=\mathbf{1}$ & $\mathrm{SV}=3$ & $\mathrm{SV}=\mathbf{5}$ & $\mathrm{CO}^{3}=1$ & $\mathrm{CO}=3$ & $\mathrm{CO}=5$ \\
\hline WG-2 & $\begin{array}{l}\text { Generation of non- } \\
\text { special waste resulting } \\
\text { from packaging and } \\
\text { surplus material in on- } \\
\text { siter municipal } \\
\text { engineering works. }\end{array}$ & $\begin{array}{l}\text { Weight of on- } \\
\text { site material } \\
{[\mathrm{kg}] .}\end{array}$ & $\mathrm{P}=0.00$ & $\begin{array}{l}0.00<\mathrm{P} \leq \\
950,618.87\end{array}$ & $\begin{array}{l}950,618.87 \\
\leq \quad \mathrm{P}< \\
22,010,443 \\
44\end{array}$ & $\begin{array}{l}\mathrm{P} \quad \geq \\
22,010,443 . \\
44\end{array}$ & & & \\
\hline WG-3 & 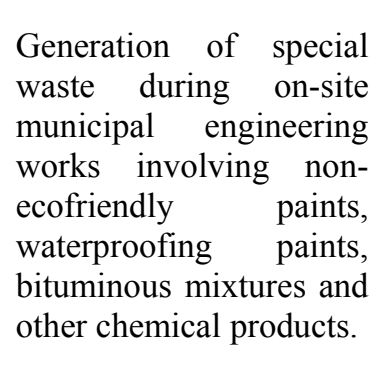 & \begin{tabular}{|lr} 
Area r paved \\
with & asphalt \\
mixture r plus \\
on-siter & surface \\
painted & with \\
non-ecofriendly \\
or \\
waterproofing \\
paints $\left[\mathrm{m}^{2}\right]$.
\end{tabular} & $\mathrm{P}=0.00$ & & $\begin{array}{l}147.53 \leq \mathrm{P} \\
<6,757.58\end{array}$ & $P \geq 6,757.58$ & \begin{tabular}{|lr} 
Selective & waste \\
collection & and delivery \\
to an & authorized \\
manager. &
\end{tabular} & 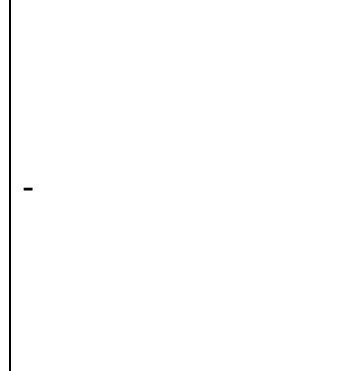 & $\begin{array}{l}\text { Non-selective waste } \\
\text { collection and delivery to } \\
\text { an authorized manager or } \\
\text { on-site waste management } \\
\text { unawareness. }\end{array}$ \\
\hline WG-4 & $\begin{array}{l}\text { Generation of municipal } \\
\text { waste by on-site } \\
\text { construction workers in } \\
\text { municipal engineering } \\
\text { works. }\end{array}$ & \begin{tabular}{|lr} 
Average & \\
number r of \\
workers per \\
day [number of \\
workers].
\end{tabular} & $\mathrm{P}=0.00$ & $\begin{array}{l}0.00<\mathrm{P} \leq \\
6.37\end{array}$ & $\begin{array}{l}6.37 \leq \mathrm{P}< \\
16.54\end{array}$ & $P \geq 16.54$ & $\begin{array}{l}\text { Selective } r \text { waste } \\
\text { collection and delivery } \\
\text { to an authorized manager } \\
\text { for future reuse or } \\
\text { recycling. }\end{array}$ & $\begin{array}{l}\text { Selective waste } \\
\text { collection and delivery } \\
\text { to an authorized } \\
\text { manager with unknown } \\
\text { final waste destination. }\end{array}$ & $\begin{array}{l}\text { Non-selective waste } \\
\text { collection and delivery to } \\
\text { an authorized manager or } \\
\text { on-site waste management } \\
\text { unawareness. }\end{array}$ \\
\hline
\end{tabular}




\begin{tabular}{|c|c|c|c|c|c|c|c|c|c|}
\hline \multicolumn{2}{|c|}{ ENVIRONMENTAL ASPECT } & INDICATOR $^{1}$ & $\mathrm{SV}^{2}=\mathbf{0}$ & $\mathrm{SV}=\mathbf{1}$ & SV $=3$ & $S V=5$ & $\mathrm{CO}^{3}=1$ & $\mathrm{CO}=3$ & $\mathrm{CO}=5$ \\
\hline \multicolumn{10}{|c|}{ SOIL ALTERATION } \\
\hline SA-1 & $\begin{array}{l}\text { Land occupancy by } \\
\text { provisional on-site } \\
\text { facilities in municipal } \\
\text { engineering works. }\end{array}$ & $\begin{array}{l}\text { Site occupation } \\
\text { of on-site } \\
\text { facilities (i.e. } \\
\text { materials and } \\
\text { waste storage } \\
\text { areas, on-site } \\
\text { machinery } \\
\text { maintenance } \\
\text { areas, etc.) } \\
{\left[\mathrm{m}^{2}\right] .}\end{array}$ & $\mathrm{P}=0.00$ & $\begin{array}{l}0< \\
80.57\end{array}$ & $\begin{array}{l}80.57 \leq \mathrm{P}< \\
132.33\end{array}$ & $P \geq 132.33$ & $\begin{array}{l}\text { The site is located in a } \\
\text { non-protected rural area } \\
\text { and the affected area is } \\
\text { located inside the site } \\
\text { perimeter or the site is } \\
\text { located in an urban area } \\
\text { and the site perimeter } \\
\text { does not affect the } \\
\text { amount of free space for } \\
\text { vehicle or pedestrian } \\
\text { circulation or the number } \\
\text { of available parking } \\
\text { places. }\end{array}$ & $\begin{array}{l}\text { The site is located in a } \\
\text { non-protected rural } \\
\text { area and the affected } \\
\text { area is located outside } \\
\text { the site perimeter. }\end{array}$ & $\begin{array}{l}\text { The site is located in an } \\
\text { area with legal protection } \\
\text { or in another area that, } \\
\text { due to its unique nature } \\
\text { (for example, its natural } \\
\text { or archaeological } \\
\text { interest), must be } \\
\text { specially protected or the } \\
\text { site is located in an urban } \\
\text { area and the site perimeter } \\
\text { invades the sidewalk, with } \\
\text { more than } 1.00 \text { m of free } \\
\text { space left for pedestrians, } \\
\text { or the site perimeter } \\
\text { affects the number of } \\
\text { available parking places } \\
\text { on the road/street, with } \\
2.75 \mathrm{~m} \text { of free space left } \\
\text { for vehicle circulation on } \\
\text { one-way roads or } 6.00 \mathrm{~m} \\
\text { on two-way roads. }\end{array}$ \\
\hline SA-2 & $\begin{array}{l}\text { Soil alteration resulting } \\
\text { from dumping during the } \\
\text { use and maintenance of } \\
\text { on-site construction } \\
\text { machinery and vehicles } \\
\text { in municipal engineering } \\
\text { works. }\end{array}$ & $\begin{array}{l}\text { Running time } \\
\text { of on-site } \\
\text { construction } \\
\text { machinery and } \\
\text { vehicles [h]. }\end{array}$ & $\mathrm{P}=0.00$ & $\begin{array}{l}0.00<\mathrm{P} \leq \\
575.04\end{array}$ & $\begin{array}{l}575.04 \leq \mathrm{P} \\
<7,083.00\end{array}$ & $P \geq 7,083.00$ & $\begin{array}{l}\text { Existence of an in situ } \\
\text { waterproof settling basin } \\
\text { or watertight tank. }\end{array}$ & $\begin{array}{l}\text { Connection to sewage } \\
\text { system, dumping in } \\
\text { septic tank and/or } \\
\text { existence of previous } \\
\text { treatment. }\end{array}$ & $\begin{array}{l}\text { Direct dumping to the } \\
\text { natural or urban } \\
\text { environment. }\end{array}$ \\
\hline
\end{tabular}




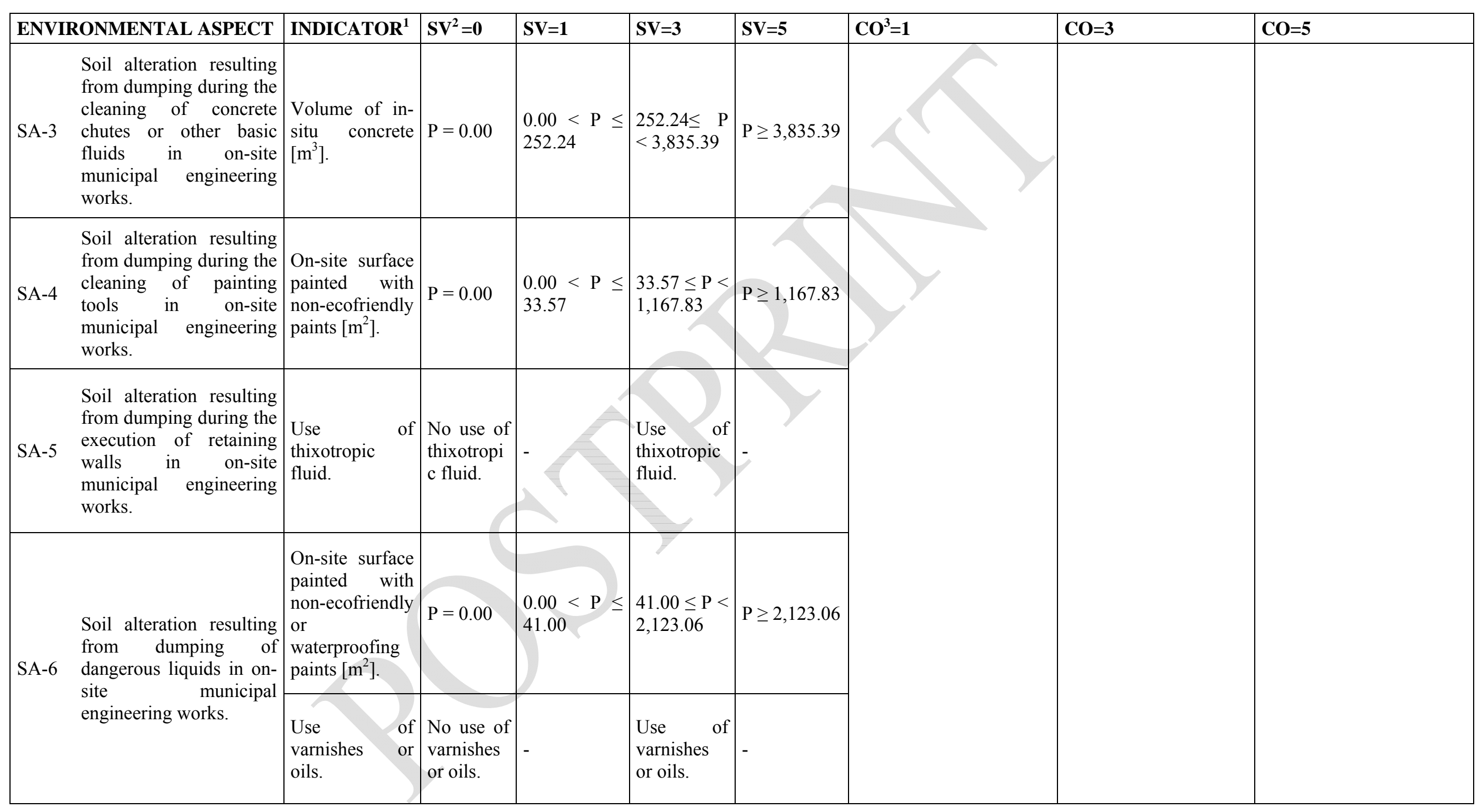




\begin{tabular}{|c|c|c|c|c|c|c|c|c|c|}
\hline \multicolumn{2}{|c|}{ ENVIRONMENTAL ASPECT } & INDICATOR $^{1}$ & $\mathrm{SV}^{2}=\mathbf{0}$ & $\mathrm{SV}=\mathbf{1}$ & $\mathrm{SV}=3$ & $\mathrm{SV}=\mathbf{5}$ & $\mathrm{CO}^{3}=1$ & $\mathrm{CO}=3$ & $\mathrm{CO}=5$ \\
\hline \multicolumn{10}{|c|}{ RESOURCES CONSUMPTION } \\
\hline RC-1 & $\begin{array}{l}\text { Water consumption to } \\
\text { avoid dust generation in } \\
\text { on-site municipal } \\
\text { engineering works. }\end{array}$ & $\begin{array}{l}\text { Water } \\
\text { consumption } \\
{\left[\mathrm{m}^{3}\right]}\end{array}$ & $\mathrm{P}=0.00$ & $\begin{array}{l}0.00<\mathrm{P} \leq \\
13.97\end{array}$ & $\begin{array}{l}13.97 \leq \mathrm{P}< \\
457.20\end{array}$ & $\mathrm{P} \geq 457.20$ & $\begin{array}{l}\text { Use of rainwater or tap } \\
\text { water. }\end{array}$ & $\begin{array}{l}\text { Use of water tankers or } \\
\text { water from rivers or } \\
\text { wells. }\end{array}$ & $\begin{array}{l}\text { Use of water from rivers } \\
\text { or wells in drought- } \\
\text { affected areas. }\end{array}$ \\
\hline $\mathrm{RC}-2$ & $\begin{array}{l}\text { Electricity consumption } \\
\text { in cutting operations } \\
\text { during on-site municipal } \\
\text { engineering works. }\end{array}$ & $\begin{array}{l}\text { Running time } \\
\text { of electrical } \\
\text { machines [h]. }\end{array}$ & $\mathrm{P}=0.00$ & $\begin{array}{l}0.00< \\
129.84\end{array}$ & $\begin{array}{l}129.84 \\
<1,047\end{array}$ & $P \geq$ & \multirow[b]{2}{*}{$\begin{array}{l}\text { Use of electricity from } \\
\text { the grid. }\end{array}$} & & \multirow[b]{2}{*}{ Use of power generators. } \\
\hline $\mathrm{RC}-3$ & $\begin{array}{l}\text { Electricity consumption } \\
\text { in on-site facilities } \\
\text { during municipal } \\
\text { engineering works. }\end{array}$ & $\begin{array}{lr}\text { Average } & \\
\text { number } & \text { of } \\
\text { workers per } \\
\text { day [number of } \\
\text { workers]. }\end{array}$ & $\mathrm{P}=0.00$ & $\begin{array}{l}0.00<\mathrm{P} \leq \\
6.37\end{array}$ & $\begin{array}{l}6.37 \leq \mathrm{P}< \\
16.54\end{array}$ & $\mathrm{P} \geq 16.54$ & & - & \\
\hline
\end{tabular}




\begin{tabular}{|c|c|c|c|c|c|c|c|c|c|}
\hline \multicolumn{2}{|c|}{ ENVIRONMENTAL ASPECT } & \multirow{2}{*}{$\begin{array}{l}\text { INDICATOR }^{1} \\
\text { Running time } \\
\text { of on-site } \\
\text { construction } \\
\text { machinery and } \\
\text { vehicles }[\mathrm{h}] .\end{array}$} & \multirow{2}{*}{\begin{tabular}{|l}
$\mathbf{S V}^{\mathbf{2}}=\mathbf{0}$ \\
$\mathrm{P}=0.00$
\end{tabular}} & \multirow{2}{*}{$\begin{array}{l}\text { SV=1 } \\
\\
0.00<\mathrm{P} \leq \\
575.04\end{array}$} & \multirow{2}{*}{\begin{tabular}{|l|}
$\mathbf{S V}=\mathbf{3}$ \\
\\
\\
$575.04 \leq \mathrm{P}$ \\
$<7,083.00$
\end{tabular}} & \multirow{2}{*}{\begin{tabular}{|l|l}
$\mathbf{S V}=5$ & $\mathbf{1}$ \\
$\mathrm{P} \geq 7,083.00$ &
\end{tabular}} & \multirow{2}{*}{ 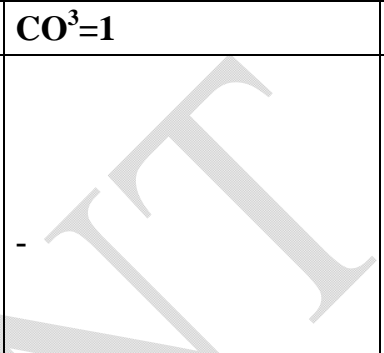 } & \multirow{2}{*}{$\begin{array}{l}\mathrm{CO}=3 \\
- \\
-\end{array}$} & \multirow{2}{*}{$\begin{array}{l}\mathbf{C O}=5 \\
\text { All cases. }\end{array}$} \\
\hline RC-4 & $\begin{array}{l}\text { Fuel consumption during } \\
\text { on-site municipal } \\
\text { engineering works. }\end{array}$ & & & & & & & & \\
\hline RC-5 & $\begin{array}{l}\text { Raw material } \\
\text { consumption during on- } \\
\text { site } \quad \text { municipal } \\
\text { engineering works. }\end{array}$ & $\begin{array}{l}\text { Weight of on- } \\
\text { site material } \\
{[\mathrm{kg}] .}\end{array}$ & $\mathrm{P}=0.00$ & $\begin{array}{l}0.00<\mathrm{P} \leq \\
950,618.87\end{array}$ & $\begin{array}{l}950,618.87 \\
\leq \quad \mathrm{P}< \\
2,010,443.4 \\
4\end{array}$ & $\begin{array}{l}\mathrm{P} \\
22,010,443 \\
44\end{array}$ & $\begin{array}{l}\text { Recycled content of raw } \\
\text { materials greater than } \\
50 \% \text {. }\end{array}$ & $\begin{array}{l}\text { Recycled content of } \\
\text { raw materials between } \\
5 \text { and } 50 \% \text {. }\end{array}$ & $\begin{array}{l}\text { Recycled content of raw } \\
\text { materials less than } 50 \% \text { or } \\
\text { it is not planned or there } \\
\text { is no information in this } \\
\text { regard. }\end{array}$ \\
\hline \multicolumn{10}{|c|}{ LOCAL ISSUES } \\
\hline LI-1 & $\begin{array}{l}\text { Generation of noise and } \\
\text { vibrations during the } \\
\text { execution of channels in } \\
\text { on-site municipal } \\
\text { engineering works. }\end{array}$ & $\begin{array}{lr}\text { Linear } & \text { metres } \\
\text { of } & \text { channels } \\
{[\mathrm{m}] .} & \end{array}$ & $P=0.00$ & $\begin{array}{l}0.00<P \leq \\
483.19\end{array}$ & $\begin{array}{l}483.19 \leq \mathrm{P} \\
< \\
13,495.93\end{array}$ & $\begin{array}{l}\mathrm{P} \\
13,495.93\end{array} \geq$ & $\begin{array}{l}\text { Isolated site or site } \\
\text { located in industrial } \\
\text { areas or areas affected } \\
\text { by noise easements. C or } \\
\text { IV-V type zones. }\end{array}$ & $\begin{array}{ll}\text { Site located in } \\
\text { residential } & \text { or } \\
\text { commercial areas. B } & \text { or } \\
\text { II-III type zones. } & \end{array}$ & $\begin{array}{l}\text { Site located in high } \\
\text { acoustic comfort areas } \\
\text { (i.e. urban areas, areas } \\
\text { near schools or hospitals, } \\
\text { areas of special ecological } \\
\text { interest, etc.). A or I type } \\
\text { zones. }\end{array}$ \\
\hline
\end{tabular}




\begin{tabular}{|c|c|c|c|c|c|c|c|c|c|}
\hline \multicolumn{2}{|c|}{ ENVIRONMENTAL ASPECT } & INDICATOR $^{1}$ & $\mathrm{SV}^{2}=\mathbf{0}$ & $S V=1$ & $\mathrm{SV}=3$ & $\mathrm{SV}=\mathbf{5}$ & \multirow[t]{2}{*}{$\mathrm{CO}^{3}=1$} & \multirow[t]{2}{*}{$\mathrm{CO}=3$} & \multirow[t]{2}{*}{$\mathrm{CO}=5$} \\
\hline LI-2 & $\begin{array}{l}\text { Generation of noise and } \\
\text { vibrations during } \\
\text { demolitions and internal } \\
\text { movements of materials } \\
\text { in on-site municipal } \\
\text { engineering works. }\end{array}$ & $\begin{array}{l}\text { Running time } \\
\text { of on-site } \\
\text { construction } \\
\text { machinery and } \\
\text { vehicles }[\mathrm{h}] .\end{array}$ & $\mathrm{P}=0.00$ & $\begin{array}{l}0.00<\mathrm{P} \leq \\
575.04\end{array}$ & $\begin{array}{l}575.04 \leq \mathrm{P} \\
<7,083.00\end{array}$ & $\mathrm{P} \geq 7,083.00$ & & & \\
\hline \multirow{2}{*}{ LI-3 } & \multirow{2}{*}{$\begin{array}{l}\text { Generation of noise and } \\
\text { vibrations during the } \\
\text { execution of earth filling } \\
\text { and paving in on-site } \\
\text { municipal engineering } \\
\text { works. }\end{array}$} & $\begin{array}{lr}\text { Volume } & \text { of } \\
\text { earth } & \text { filling } \\
{\left[\mathrm{m}^{3}\right] .} & \end{array}$ & $\mathrm{P}=0.00$ & $\begin{array}{l}0.00<\mathrm{P} \leq \\
306.48\end{array}$ & $\begin{array}{l}306.48 \leq \mathrm{P} \\
< \\
15,819.11\end{array}$ & $\begin{array}{l}\mathrm{P} \\
15,819.11\end{array}$ & & & \\
\hline & & $\begin{array}{l}\text { Paved area } \\
{\left[\mathrm{m}^{2}\right] .}\end{array}$ & $\mathrm{P}=0.00$ & $\begin{array}{l}0.00<\mathrm{P} \\
560.25\end{array}$ & $\begin{array}{l}560.25 \leq P \\
< \\
12,317.47\end{array}$ & $\begin{array}{l}\mathrm{P} \\
12,317.47\end{array}$ & & & \\
\hline & \multirow{2}{*}{$\begin{array}{l}\text { Generation of smells } \\
\text { during painting and } \\
\text { paving in on-site } \\
\text { municipal engineering } \\
\text { works. }\end{array}$} & $\begin{array}{l}\text { Paved area with } \\
\text { asphalt mixture } \\
{\left[\mathrm{m}^{2}\right] .}\end{array}$ & $\mathrm{P}=0.00$ & $\begin{array}{l}0.00<\mathrm{P} \leq \\
92.66\end{array}$ & $\begin{array}{l}92.66 \leq \mathrm{P}< \\
8,171.42\end{array}$ & $\mathrm{P} \geq 8,171.42$ & \multirow{2}{*}{$\begin{array}{l}\text { Isolated } \\
\text { located } \\
\text { areas. }\end{array}$} & \multirow{2}{*}{$\begin{array}{ll}\text { Site located in } & \text { in } \\
\text { residential } & \text { or } \\
\text { commercial areas or in } \\
\text { a non-protected rural } \\
\text { area. }\end{array}$} & \multirow{2}{*}{$\begin{array}{l}\text { Site located in an area } \\
\text { with legal protection, or in } \\
\text { another area that, due to } \\
\text { its unique nature (for } \\
\text { example, its natural or } \\
\text { archaeological interest), } \\
\text { must be specially } \\
\text { protected. }\end{array}$} \\
\hline & & $\begin{array}{l}\text { On-site surface } \\
\text { painted with } \\
\text { non-ecofriendly } \\
\text { paints }\left[\mathrm{m}^{2}\right] .\end{array}$ & $\mathrm{P}=0.00$ & $\begin{array}{l}0.00<\mathrm{P} \leq \\
33.57\end{array}$ & $\begin{array}{l}33.57 \leq \mathrm{P}< \\
1,167.83\end{array}$ & $\mathrm{P} \geq 1,167.83$ & & & \\
\hline
\end{tabular}




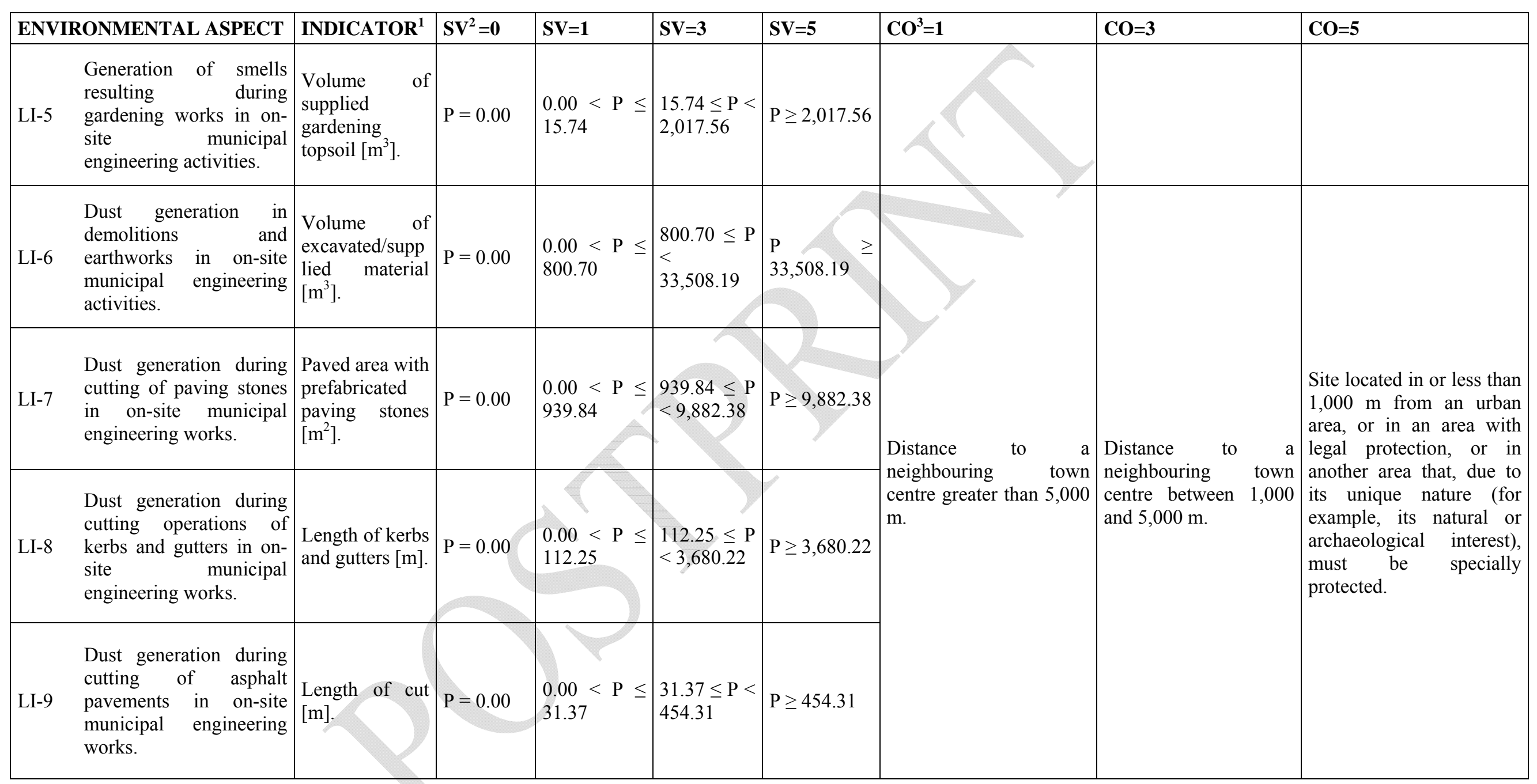




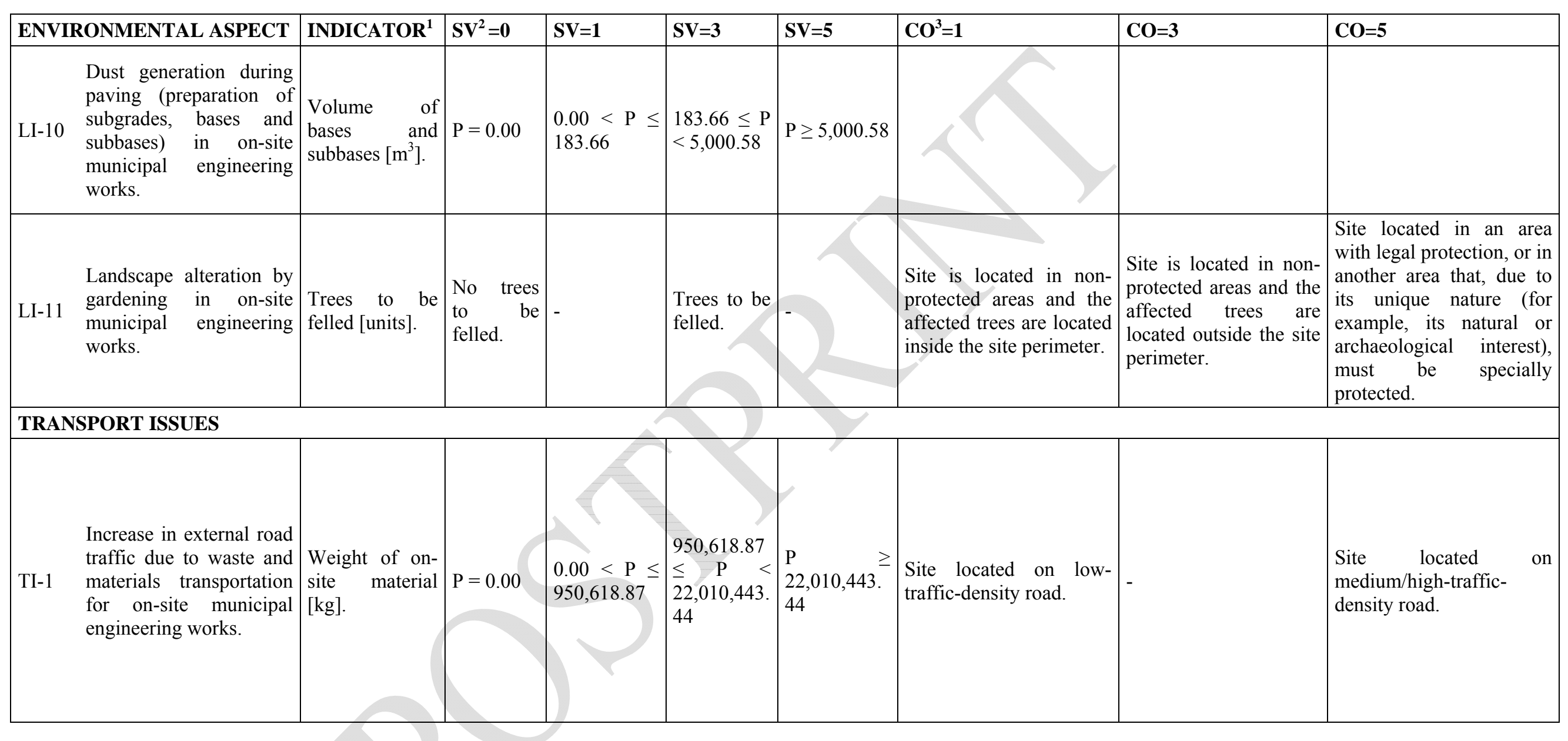




\begin{tabular}{|c|c|c|c|c|c|c|c|c|c|}
\hline \multicolumn{2}{|c|}{ ENVIRONMENTAL ASPECT } & INDICATOR $^{1}$ & $\mathrm{SV}^{2}=\mathbf{0}$ & $\mathrm{SV}=1$ & $S V=3$ & $S V=5$ & $\mathrm{CO}^{3}=1$ & $\mathrm{CO}=3$ & \multirow{2}{*}{\begin{tabular}{|l|}
$\mathbf{C O}=\mathbf{5}$ \\
\\
\\
Site located on \\
medium/high-traffic- \\
density road, with less \\
than $2.75 \mathrm{~m}$ of free space \\
left for vehicle circulation \\
on one-way roads, or less \\
than $6.00 \mathrm{~m}$ on two-way \\
roads.
\end{tabular}} \\
\hline TI-2 & $\begin{array}{l}\text { Interference in external } \\
\text { road traffic due to the } \\
\text { transport of large-size } \\
\text { elements for on-site } \\
\text { municipal engineering } \\
\text { works. }\end{array}$ & $\begin{array}{l}\text { Number of } \\
\text { units longer } \\
\text { than } 12.00 \mathrm{~m} \text { to } \\
\text { be transported } \\
\text { [units]. }\end{array}$ & $\begin{array}{l}\text { No units } \\
\text { longer } \\
\text { than } 12.00 \\
\mathrm{~m} \text { to be } \\
\text { transporte } \\
\text { d. }\end{array}$ & - & $\begin{array}{l}\text { Units } \\
\text { longer than } \\
12.00 \mathrm{~m} \text { to } \\
\text { be } \\
\text { transported. }\end{array}$ & - & $\begin{array}{l}\text { Site located on low- } \\
\text { traffic-density road. }\end{array}$ & $\begin{array}{l}\text { Site located on } \\
\text { medium/high-traffic- } \\
\text { density road with } 2.75 \\
\text { m of free space left for } \\
\text { vehicle circulation on } \\
\text { one-way roads, or } 6.00 \\
\text { m on two-way roads. }\end{array}$ & \\
\hline \multicolumn{10}{|c|}{ ENVIRONMENTAL ACCIDENTS } \\
\hline EA-1 & $\begin{array}{l}\text { Environmental accidents } \\
\text { during underground } \\
\text { channel execution } \\
\text { (drains, sewage systems } \\
\text { and services) in on-site } \\
\text { municipal engineering } \\
\text { works. }\end{array}$ & 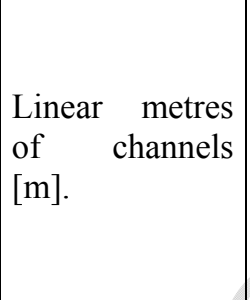 & $\mathrm{P}=0.00$ & $\begin{array}{l}0.00<\mathrm{P} \leq \\
483.19\end{array}$ & $\begin{array}{l}483.19 \leq \mathrm{P} \\
< \\
13,495.93\end{array}$ & ${ }_{13,495.93}^{P} \geq$ & \multirow{2}{*}{$\begin{array}{l}\text { Isolated site (distance to } \\
\text { nearby occupied } \\
\text { buildings, forested areas } \\
\text { or other high-fire-risk } \\
\text { areas is greater than } 500 \\
\mathrm{~m}) \text {. }\end{array}$} & \multirow{2}{*}{$\begin{array}{l}\text { Site is located in a non- } \\
\text { protected area and the } \\
\text { distance to nearby } \\
\text { occupied buildings, } \\
\text { forested areas or other } \\
\text { high-fire-risk areas is } \\
\text { between } 100 \text { and } 500 \\
\text { m. }\end{array}$} & \multirow{2}{*}{$\begin{array}{l}\text { Site is located in an area } \\
\text { with legal protection, or in } \\
\text { another area that, due to } \\
\text { its unique nature (for } \\
\text { example, its natural or } \\
\text { archaeological interest) } \\
\text { must be protected or it is } \\
\text { located in a non-protected } \\
\text { area and the distance to } \\
\text { nearby occupied } \\
\text { buildings, forested areas } \\
\text { or other high-fire-risk } \\
\text { areas is less than } 100 \mathrm{~m} \text {. }\end{array}$} \\
\hline EA-2 & $\begin{array}{l}\text { Environmental accidents } \\
\text { during demolitions, } \\
\text { earthworks and soil } \\
\text { conditioning in on-site } \\
\text { municipal engineering } \\
\text { works. }\end{array}$ & $\begin{array}{l}\text { Volume of } \\
\text { excavated/supp } \\
\text { lied material } \\
{\left[\mathrm{m}^{3}\right]}\end{array}$ & $P=0.00$ & $\begin{array}{l}0.00<\mathrm{P} \leq \\
800.70\end{array}$ & $\begin{array}{l}800.70 \leq \mathrm{P} \\
< \\
33,508.19\end{array}$ & $\begin{array}{l}\mathrm{P} \\
33,508.19\end{array}$ & & & \\
\hline
\end{tabular}




\begin{tabular}{|c|c|c|c|c|c|c|c|c|c|}
\hline \multicolumn{2}{|c|}{ ENVIRONMENTAL ASPECT } & INDICATOR $^{1}$ & $\mathrm{SV}^{2}=\mathbf{0}$ & SV=1 & $\mathrm{SV}=3$ & $\mathrm{SV}=\mathbf{5}$ & $\mathrm{CO}^{3}=1$ & $\mathrm{CO}=3$ & $\mathrm{CO}=5$ \\
\hline EA-3 & $\begin{array}{l}\text { Environmental accidents } \\
\text { caused by fires in } \\
\text { machinery in on-site } \\
\text { municipal engineering } \\
\text { works. }\end{array}$ & $\begin{array}{l}\text { Running time } \\
\text { of on-site } \\
\text { construction } \\
\text { machinery and } \\
\text { vehicles }[\mathrm{h}] .\end{array}$ & $\mathrm{P}=0.00$ & $\mid \begin{array}{l}0.00<\mathrm{P} \leq \\
575.04\end{array}$ & $\begin{array}{l}575.04 \leq \mathrm{P} \\
<7,083.00\end{array}$ & $P \geq 7,083.00$ & & & \\
\hline EA-4 & $\begin{array}{l}\text { Environmental accidents } \\
\text { caused by fires in } \\
\text { storage areas in on-site } \\
\text { municipal engineering } \\
\text { works. }\end{array}$ & $\begin{array}{l}\text { Weight of on- } \\
\text { site material } \\
{[\mathrm{kg}] .}\end{array}$ & $\mathrm{P}=0.00$ & $\mid \begin{array}{l}0.00<\mathrm{P} \leq \\
950,618.87\end{array}$ & $\begin{array}{l}950,618.87 \\
\leq \quad P \quad< \\
22,010,443 \\
44\end{array}$ & & & & \\
\hline \multicolumn{10}{|c|}{ EFFECTS ON BIODIVERSITY } \\
\hline EB-1 & $\begin{array}{l}\text { Effects on biodiversity } \\
\text { due to edaphic soil loss } \\
\text { or soil erosion during } \\
\text { earthworks in on-site } \\
\text { municipal engineering } \\
\text { activities. }\end{array}$ & $\begin{array}{l}\text { Volume of } \\
\text { excavated } \\
\text { material }\left[\mathrm{m}^{3}\right] .\end{array}$ & $\mathrm{P}=0.00$ & $\begin{array}{l}0.00 \\
416 .\end{array}$ & $\begin{array}{l}416.49 \leq \mathrm{P} \\
< \\
19,831.43\end{array}$ & $\begin{array}{l}\mathrm{P} \\
19,831.43\end{array}$ & $\begin{array}{l}\text { Site is located in non- } \\
\text { protected areas and the } \\
\text { affected area is located } \\
\text { inside the site perimeter } \\
\text { or outside the site } \\
\text { perimeter when there is } \\
\text { no edaphic soil. }\end{array}$ & $\begin{array}{l}\text { Site is located in non- } \\
\text { protected areas and the } \\
\text { affected area is located } \\
\text { outside the site } \\
\text { perimeter when there is } \\
\text { edaphic soil. }\end{array}$ & $\begin{array}{l}\text { Areas with legal } \\
\text { protection or other areas } \\
\text { that, due to their nature } \\
\text { (for example, their natural } \\
\text { or archaeological } \\
\text { interest), must be } \\
\text { specially protected. }\end{array}$ \\
\hline EB-2 & $\begin{array}{l}\text { Effects on biodiversity } \\
\text { due to vegetation loss in } \\
\text { on-site r municipal } \\
\text { engineering works. }\end{array}$ & $\begin{array}{l}\text { Trees to be } \\
\text { felled [units]. }\end{array}$ & $\begin{array}{l}\text { No trees } \\
\text { to r be } \\
\text { felled. }\end{array}$ & & $\begin{array}{l}\text { Trees to be } \\
\text { felled. }\end{array}$ & - & $\begin{array}{l}\text { Site is located in non- } \\
\text { protected areas and the } \\
\text { affected area is located } \\
\text { inside the site perimeter } \\
\text { or outside the site } \\
\text { perimeter when there is } \\
\text { no vegetation. }\end{array}$ & $\begin{array}{l}\text { Site is located in non- } \\
\text { protected areas and the } \\
\text { affected area is located } \\
\text { outside the site } \\
\text { perimeter when there is } \\
\text { vegetation. }\end{array}$ & $\begin{array}{l}\text { Areas with legal } \\
\text { protection or other areas } \\
\text { that, due to their nature } \\
\text { (for example, their natural } \\
\text { or archaeological } \\
\text { interest), must be } \\
\text { specially protected. }\end{array}$ \\
\hline
\end{tabular}




\begin{tabular}{|l|l|l|l|l|l|l|l|}
\hline ENVIRONMENTAL ASPECT & INDICATOR $^{1}$ & $\mathbf{S V}^{2}=\mathbf{0}$ & $\mathbf{S V}=\mathbf{1}$ & $\mathbf{S V}=\mathbf{3}$ & $\mathbf{S V}=\mathbf{5}$ & $\mathbf{C O}^{\mathbf{3}}=\mathbf{1}$ & $\mathbf{C O}=\mathbf{3}$ \\
\hline & $\begin{array}{l}\text { Area } \\
\text { vegetation of } \\
\text { clearing }\left[\mathrm{m}^{2}\right] .\end{array}$ & $\mathrm{P}=0.00$ & $\begin{array}{l}0.00<\mathrm{P} \leq \\
91.97\end{array}$ & $\begin{array}{l}91.97 \leq \mathrm{P}< \\
24,372.16\end{array}$ & $\begin{array}{l}\mathrm{P} \\
24,372.16\end{array}$ & & \\
\end{tabular}

${ }^{1} \mathrm{P}$ : indicator for measuring the severity of environmental impacts (extracted from the quantitative data in the project documents). ${ }^{2} \mathrm{SV}$ : severity of the environmental impact.

${ }^{3} \mathrm{CO}$ : concerns of interested parties.

Table 1. Evaluation of the environmental impacts of municipal engineering works. 


\subsection{Assessment of the on-site environmental aspects of municipal engineering projects}

During the process of identifying environmental aspects of on-site municipal engineering works, we only analysed environmental criteria that were dependant on the on-site construction stages and activities and not on the municipal engineering project (scale, probability and duration of the impact). The remaining components of significance (those that depended on each specific project) were examined in this stage. The severity parameter (SV) estimates the magnitude (or relevance) of each environmental aspect in quantitative terms (Gangolells et al., 2009). The concerns parameter (CO) includes the concerns of internal and external stakeholders who may perceive that certain environmental impacts are highly significant. Internal interested parties include neighbouring communities that are directly affected by a proposed project, whereas external interested parties comprise society as a whole and are represented by community associations, environmentalists, non-governmental organizations and the media, among others (Gangolells et al., 2011).

We developed a four-point scale that includes detailed criteria to help determine whether parameters are significant (Gangolells et al., 2009; 2011). We also assigned numerical limits to the categories. In terms of impact severity, a score of " 0 " indicated no impact, "1" represented an environmental impact of low severity, "3" referred to an environmental impact of moderate severity and " 5 " indicated that the impact severity was major. Scores "1", "3" and "5" were assigned to little/no concern to interested parties, secondary concern to all or most interested parties and primary concern to all or most interested parties, respectively.

Thus, according to Gangolells et al. (2011), the significance of an environmental impact in a particular municipal engineering project is obtained using the following expression:

$$
S G_{E j}=S V_{j} \cdot C O_{j}
$$

where $\mathrm{SG}_{\mathrm{Ej}}$ designates the significance of a particular environmental impact $\mathrm{j}$ in a specific municipal engineering project, $\mathrm{SV}_{\mathrm{j}}$ denotes the impact severity, and $\mathrm{CO}_{\mathrm{j}}$ corresponds to the concerns parameter (Table 2). 


\begin{tabular}{|c|c|c|c|c|c|}
\hline & \multicolumn{4}{|c|}{$\begin{array}{c}\text { SEVERITY OF ENVIRONMENTAL } \\
\text { IMPACTS } \\
\left(\mathrm{SV}_{\mathrm{j}}\right)\end{array}$} \\
\hline & & $\begin{array}{c}\text { Non- } \\
\text { existent }\end{array}$ & $\begin{array}{c}\text { Low } \\
\text { severity }\end{array}$ & $\begin{array}{c}\text { Moderate } \\
\text { severity }\end{array}$ & $\begin{array}{c}\text { Major } \\
\text { severity }\end{array}$ \\
\hline \multirow{3}{*}{ 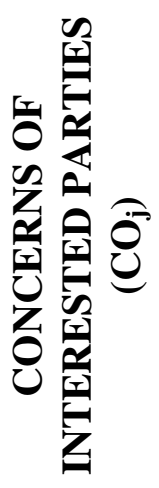 } & $\begin{array}{l}\text { Little/no concern to } \\
\text { interested parties }\end{array}$ & 0 & 1 & 3 & 9 \\
\hline & $\begin{array}{l}\text { Secondary concern to } \\
\text { all or most interested } \\
\text { parties }\end{array}$ & 0 & 3 & 9 & 15 \\
\hline & $\begin{array}{l}\text { Primary concern to all } \\
\text { or most interested } \\
\text { parties }\end{array}$ & 0 & 5 & & 25 \\
\hline
\end{tabular}

Table 2. Significance matrix for assessing the on-site environmental impacts of municipal engineering works.

Thus, the significance of an environmental impact depends on both its severity and the corresponding concerns of interested parties. For example, given a particular level of severity, the significance of an environmental accident during demolitions, earthworks and soil conditioning will depend on the site location. The significance will be minimum if the site is isolated, which means that the distance to the nearest occupied building, forested areas or other high-fire-risk areas is greater than $500 \mathrm{~m}$. The significance of the impact will be greater if the site is located in a non-protected area and the distance to the nearest occupied buildings, forested areas or other high-risk areas is between 100 and $500 \mathrm{~m}$. Finally, the significance of the impact will be maximum if the site is located in an area with legal protection or in another area that, due to its unique nature (for example, its natural or archaeological interest) must be protected or when the site is located in a non-protected area and the distance to the nearest occupied buildings, forested areas or other high-risk areas is less than $100 \mathrm{~m}$. This is exemplified in the matrix for the assessment of on-site environmental impacts of municipal engineering works (Table 1).

The level of acceptability of a potential environmental impact is set as an intermediate situation involving moderate severity $\left(\mathrm{SV}_{\mathrm{j}}=3\right)$ of secondary concern to all or most interested parties $\left(\mathrm{CO}_{\mathrm{j}}=3\right)$. If an environmental impact is found to be significant $\left(\mathrm{SG}_{\mathrm{j}}>9\right)$ in the assessment, a range of measures for mitigating the adverse impact should be implemented during on-site activities.

\subsubsection{Development of indicators}

In order to ensure the objectivity of the assessment process, indicators must be based on 
specific observable or measurable characteristics of the project being assessed. Bearing in mind that this data should be available during the construction planning stage, indicators must be based on information contained in municipal engineering project documents (i.e. the building specifications, the drawings, the bill of quantities, the health and safety plan, and the budget). Consequently, we used the content analysis method, which is a classical approach to research problems that is based on documentary evidence (Holsti, 1969; Rattleff, 2007; Shen et al., 2011). Suitable direct or indirect indicators were proposed, depending on their availability in the project documents, to assess each environmental impact of on-site municipal engineering works in terms of severity and concerns of interested parties. Direct indicators were preferred, as they are unequivocal. For example, quantity of water (expressed in $\mathrm{m}^{3}$ ) is a good direct environmental indicator of the severity of the environmental aspect RC-1 "Water consumption to avoid dust generation in on-site municipal engineering works", which is included in the 'resource consumption' category. This parameter can easily be found in the project's bill of quantities. In some cases, environmental impacts cannot be directly measured through project documents or the process is extremely time-consuming. In these cases, indirect indicators are proposed. Indirect indicators ensure the objectivity of the evaluation process, and provide an admissible order of magnitude. For example, the amount of on-site surfaces painted with non-ecofriendly paints (expressed in $\mathrm{m}^{2}$ ) is a good indirect indicator of the severity of environmental aspect SA-4 "Soil alteration resulting from dumping during cleaning of painting tools in on-site municipal engineering works". This parameter can also be obtained from the bill of quantities. As far as possible, quantitative indicators are proposed. However, in some cases, qualitative indicators have to be used, especially in the assessment of concerns of interested parties as related numerical data is generally not available in the pre-construction stage. For example, the indicator for assessing concerns of interested parties in about environmental aspect LI-1 "Generation of noise and vibrations during the execution of channels in on-site municipal engineering works" is related to the location of the project and distinguishes between (1) isolated sites or sites in industrial areas and areas affected by noise easements, (2) sites located in residential or commercial areas and (3) sites located in high acoustic comfort areas (for example, urban areas, areas near schools or hospitals and areas of special ecological interest). Table 1 shows the developed indicators.

\subsubsection{Determination of significance limits}

In order to achieve an even outcome in assessments of impact severity and concerns of interested parties, we developed a matrix model with corresponding indicators and limits for the categories (Table 1). To assess the severity of the environmental aspects, we carried out a statistical analysis of the quantitative indicators of 25 new-build and remodelling municipal engineering projects. These projects were for areas ranging from 682 to $47,842 \mathrm{~m}^{2}$, with a total project cost of $€ 188,130$ to $€ 9,411,009$ (Table 3). According to Gangolells et al. (2009), the assumption is that a high proportion of municipal engineering projects have a marginally significant impact. To establish the upper and lower limits of marginally significant impacts, a $68 \%$ confidence interval $[\mu-$ $\sigma, \mu+\sigma]$ was calculated for each indicator (Gangolells et al., 2009). If the indicator is lower than $\mu-\sigma$, the severity of the environmental aspect is considered to be low, 
whereas if it is higher than $\mu+\sigma$, the severity of the environmental aspect is considered to be major. Indicators in between are considered to reflect moderate severity. For each of the quantitative indicators considered in the model, Table 4 summarizes the corresponding lower and upper limits of the $68 \%$ confidence interval, as well as the means and standard deviations of the corresponding distributions. In accordance with Gangolells et al. (2011), assessment scales for non-quantitative indicators were described with greater care and precision, to avoid relying on subjective judgements. Table 1 includes the assessment scales for both quantitative and qualitative indicators.

\begin{tabular}{|c|c|c|c|}
\hline PROJECT & DESCRIPTION & $\begin{array}{c}\text { INTERVENTION } \\
\text { AREA }\left[\mathrm{m}^{2}\right]\end{array}$ & \begin{tabular}{|r|} 
TOTAL \\
PROJECT \\
COST [€] \\
\end{tabular} \\
\hline 1 & Urban area redevelopment & 15,973 & $9,411,009$ \\
\hline 2 & Redevelopment of a street & 682 & $1,730,163$ \\
\hline 3 & Development of streets & 6,610 & 799,435 \\
\hline 4 & Development of a street & 1,391 & 572,510 \\
\hline 5 & Development of a park & 23,236 & $1,747,931$ \\
\hline 6 & Development of streets & 7,800 & $1,373,225$ \\
\hline 7 & Redevelopment of streets & 2,940 & 538,553 \\
\hline 8 & Redevelopment of a square & 2,795 & 623,322 \\
\hline 9 & Redevelopment of a square and streets & 2,230 & 358,789 \\
\hline 10 & Redevelopment of a square & 7,727 & 316,465 \\
\hline 11 & $\begin{array}{l}\text { Redevelopment of streets and } \\
\text { construction of roundabout }\end{array}$ & 1,332 & 188,130 \\
\hline 12 & Redevelopment of a square & 2,879 & 419,683 \\
\hline 13 & Development of a street & 5,232 & 454,834 \\
\hline 14 & Redevelopment of an avenue & 17,830 & $1,759,514$ \\
\hline 15 & Redevelopment of a square & 2,820 & 484,152 \\
\hline 16 & Development of squares & 7,907 & $1,751,294$ \\
\hline 17 & Development of streets & 4,552 & 578,231 \\
\hline 18 & Development of streets & 3,671 & 934,140 \\
\hline 19 & Development of an industrial sector & 9,178 & $1,423,655$ \\
\hline 20 & Neighbourhood redevelopment & 20,700 & $1,904,500$ \\
\hline 21 & Street and square redevelopment & 2,983 & 334,589 \\
\hline
\end{tabular}




\begin{tabular}{|l|l|l|l|}
\hline 22 & Campsite development & 47,842 & $3,472,784$ \\
\hline 23 & Redevelopment of streets & 3,009 & 751,078 \\
\hline 24 & Land development & 55,598 & $2,331,177$ \\
\hline 25 & Development of streets & 7,252 & $1,167,753$ \\
\hline
\end{tabular}

Table 3. Main characteristics of the municipal engineering projects. 


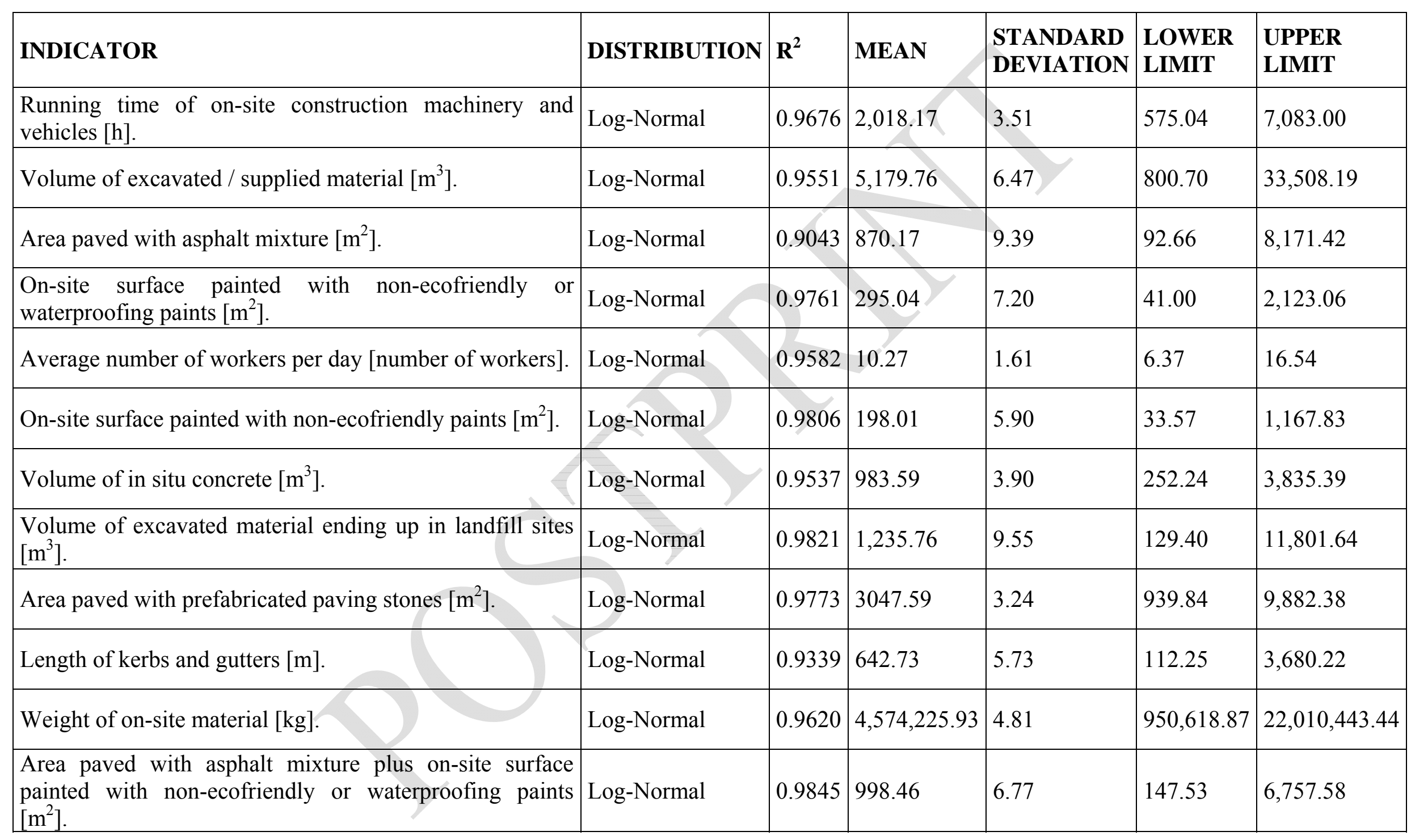




\begin{tabular}{|c|c|c|c|c|c|c|}
\hline INDICATOR & DISTRIBUTION & $\mathbf{R}^{2}$ & MEAN & $\begin{array}{l}\text { STANDARD } \\
\text { DEVIATION }\end{array}$ & $\begin{array}{l}\text { LOWER } \\
\text { LIMIT }\end{array}$ & $\begin{array}{l}\text { UPPER } \\
\text { LIMIT }\end{array}$ \\
\hline Site occupation of on-site facilities $\left[\mathrm{m}^{2}\right]$. & Log-Normal & 0.9497 & 103.25 & 1.28 & 80.57 & 132.33 \\
\hline Water consumption $\left[\mathrm{m}^{3}\right]$. & Log-Normal & 0.9664 & 79.91 & 5.72 & 13.97 & 457.20 \\
\hline Running time of electrical machines [h]. & Log-Normal & 0.9325 & 368.77 & 2.84 & 129.84 & $1,047.43$ \\
\hline Volume of earth filling $\left[\mathrm{m}^{3}\right]$. & Log-Normal & 0.9556 & $2,201.89$ & 7.18 & 306.48 & $15,819.11$ \\
\hline Paved area $\left[\mathrm{m}^{2}\right]$. & Log-Normal & 0.9443 & $2,626.94$ & 4.69 & 560.25 & $12,317.47$ \\
\hline Volume of bases and subbases $\left[\mathrm{m}^{3}\right]$. & Log-Normal & 0.9635 & 958.35 & 5.22 & 183.66 & $5,000.58$ \\
\hline Volume of excavated material $\left[\mathrm{m}^{3}\right]$ & Log-Normal & 0.9502 & $2,873.95$ & 6.90 & 416.49 & $19,831.43$ \\
\hline Area of vegetation clearing $\left[\mathrm{m}^{2}\right]$. & Log-Normal & 0.9518 & $1,497.21$ & 16.28 & 91.97 & $24,372.16$ \\
\hline
\end{tabular}

Table 4. Statistical analysis for quantitative indicators. 


\subsubsection{Determination of the overall environmental impact of a municipal engineering project}

The model assesses the overall environmental impact level of a municipal engineering project, as shown in (3).

$$
R_{E}=\sum_{j=1}^{n} S G_{E j}
$$

where $R_{E}$ denotes the overall environmental impact level of a municipal engineering project and $\mathrm{SG}_{\mathrm{Ej}}$ designates the significance of a particular environmental impact $\mathrm{j}$ in a specific municipal engineering project.

The municipal engineering project with the highest sum is considered to have the most significant environmental impact. As predictions must always be made for the worst scenario (Toro et al., 2010), if the documents of a municipal engineering project lack the information needed to assess a particular environmental aspect, its severity or the concerns of interested parties are automatically assumed to have the highest value $\left(\mathrm{SV}_{\mathrm{j}}=5\right.$ or $\left.\mathrm{CO}_{\mathrm{j}}=5\right)$. When an environmental aspect is assessed through two or more indicators, only the highest value is taken into account in the overall environmental impact level.

\section{Case study}

In this section, we apply the model to two case studies, to illustrate its practical application and to demonstrate how significant on-site environmental impacts of municipal engineering projects can be highlighted in advance.

The first case study is a square remodelling project that involves work on an area of $2,802.83 \mathrm{~m}^{2}$ and has a total project cost of $€ 628,547.57$. Initially developed during the forties, the square is located in a high acoustic comfort area of Barcelona, close to a hospital. Bounded on the west and south by buildings, the site is adjacent to a medium traffic density road to the east and a street to the north (Figure 1). The project aims to rationalize the public space by defining a clearer road layout, which will give priority to pedestrians and improve accessibility to disabled people. The project also aims to update and strengthen the capacity of the existing aerial and underground urban public facilities and utilities. The street lighting will be fully renovated and existing overhead electric cables will be placed underground. In general, the sewer system will be maintained, although some conduits and access chambers will be rebuilt. Two new storm drains and corresponding manholes are also foreseen in the project. The existing pavement made of cobbles will be replaced by granite paving slabs. In order to improve rainwater collection, existing slopes will be corrected. An asphalt road surface will be used to delimit road traffic. The existing arboreal vegetation will be maintained, but it will be rearranged according to the new design. Three new linear corten steel flower beds are planned to reduce the visual impact of road traffic. Existing street furniture will also be fully renovated. According to the Health and Safety Plan, the site perimeter will 
invade the pavement, but $1.5 \mathrm{~m}$ of free space will be left for pedestrians. The two-way road will not be affected by the construction site. Since the project is located in an urban area, the existing water supply, electrical grid and sewage system will be used during on-site construction works. Construction project documents do not include information about the recycled content of raw materials. The documents do plan for selective waste collection and later delivery to an authorized manager, although the final waste destination is not explicitly stated.

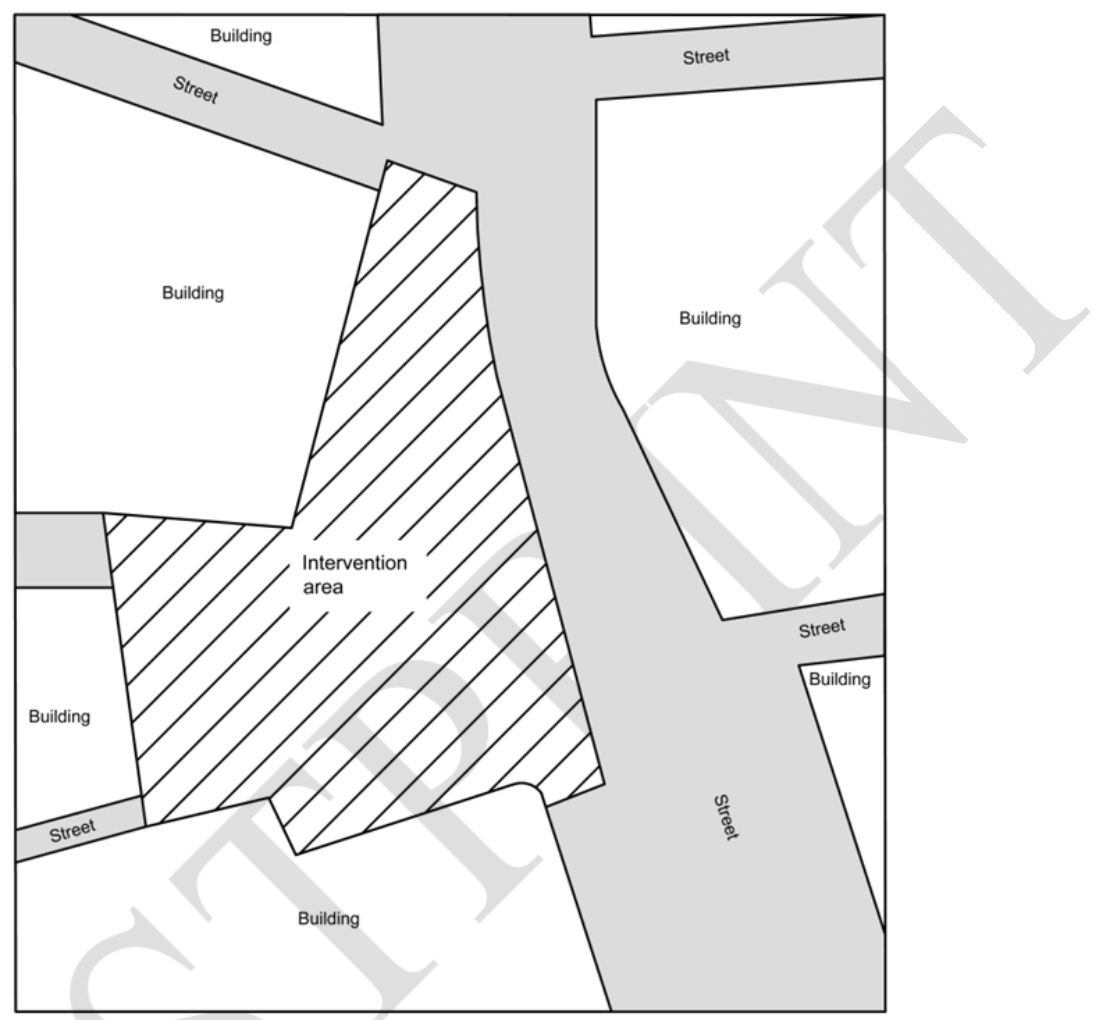

Figure 1. Schematic drawing of case study 1.

The second case study is a $73,000 \mathrm{~m}^{2}$ site that will be developed for 63 new dwellings. The project has a total cost of $€ 2,650,455.65$. The main characteristics of this case study are shown in Table 5. The site is located within a residential area of a city in the Barcelona metropolitan area. The site is surrounded by low traffic density streets and other nearby occupied buildings (Figure 2). On-site works include vegetation clearance (some trees inside the site perimeter will have to be felled), earthworks, the installation of a separate sewerage system, a telecommunication network, an electricity supply network, a water supply network, a gas supply network and street lighting, paving, gardening and signposting. The main streets are asphalted and have paved sidewalks (streets A, D, E and part of streets G and B), whereas all the other streets are totally covered with paving stones. Paving stones are also used for steps. A separate sewerage system is foreseen in the project. The storm drainage system will collect storm water, whereas the sanitary sewerage system will collect domestic sewage. In one-way streets, the herringbone method is used for street lighting. In this case, the distance between streetlights is $19 \mathrm{~m}$. In two-way streets, a bilateral distribution is used and the distance 
between lights ranges from $14 \mathrm{~m}$ to $22 \mathrm{~m}$. The public utility networks, including telecommunications, electricity, water and gas, are dimensioned to serve 63 dwellings. According to the project documents, the site will not affect the surrounding public space. The project documents plan in situ reuse of earth waste, including topsoil, and selective waste collection and delivery to an authorized manager for future reuse or recycling. However, the recycled content of raw materials is not planned. Existing surrounding networks will provide electricity, water and sewage disposal during on-site works.

Table 6 shows the detailed assessment results for these case studies. 


\begin{tabular}{|c|c|c|c|c|c|c|c|}
\hline STREET & $\begin{array}{c}\text { LENGTH } \\
{[\mathrm{m}]}\end{array}$ & $\begin{array}{c}\text { WIDTH } \\
\text { [m] }\end{array}$ & $\begin{array}{c}\text { NUMBER AND } \\
\text { WIDTH OF } \\
\text { ROADS [m] }\end{array}$ & $\begin{array}{c}\text { NUMBER AND } \\
\text { WIDTH OF } \\
\text { PAVEMENTS } \\
{[\mathrm{m}]}\end{array}$ & $\begin{array}{c}\text { NUMBER } \\
\text { AND WIDTH } \\
\text { OF PARKING } \\
\text { AREAS [m] }\end{array}$ & $\begin{array}{l}\text { NUMBER OF } \\
\text { FLOWER } \\
\text { BEDS }\end{array}$ & NOTES \\
\hline A & 152.15 & $\begin{array}{l}9.30- \\
10.50 \\
\end{array}$ & $1 / 3.50$ & $2 / 2.25-3.60$ & $0-1 / 2.00$ & 0 & - \\
\hline B & 133.42 & $\approx 8.00$ & $1 / 3.50$ & $2 / 2.25-2.80$ & 0 & 1 & - \\
\hline $\mathrm{C}$ & 69.00 & 8.50 & $1 / 3.50$ & $2 / 1.75-2.25$ & 0 & 1 & - \\
\hline $\mathrm{D}$ & 314.14 & 9.55 & $1 / 3.50$ & $2 / 2.80-2.25$ & 0 & 1 & - \\
\hline $\mathrm{E}$ & 22.70 & 9.55 & $1 / 3.50$ & $2 / 2.80-2.25$ & 0 & 1 & - \\
\hline $\mathrm{F}$ & 87.30 & 10.60 & $2 / 3.00$ & $2 / 2.10-2.50$ & 0 & 0 & - \\
\hline G & 176.64 & $\begin{array}{l}7.50- \\
9.00 \\
\end{array}$ & $1 / 3.50$ & $2 / 1.60-2.80$ & 0 & 1 & - \\
\hline $\mathrm{H}$ & 117.60 & 6.10 & 0 & 0 & 0 & 0 & $\begin{array}{l}\text { Steep slope solved } \\
\text { with steps. }\end{array}$ \\
\hline I & 66.00 & 5.00 & 0 & 0 & 0 & 0 & $\begin{array}{l}\text { Steep slope solved } \\
\text { with steps. }\end{array}$ \\
\hline
\end{tabular}

Table 5. Main characteristics of the second case study. 


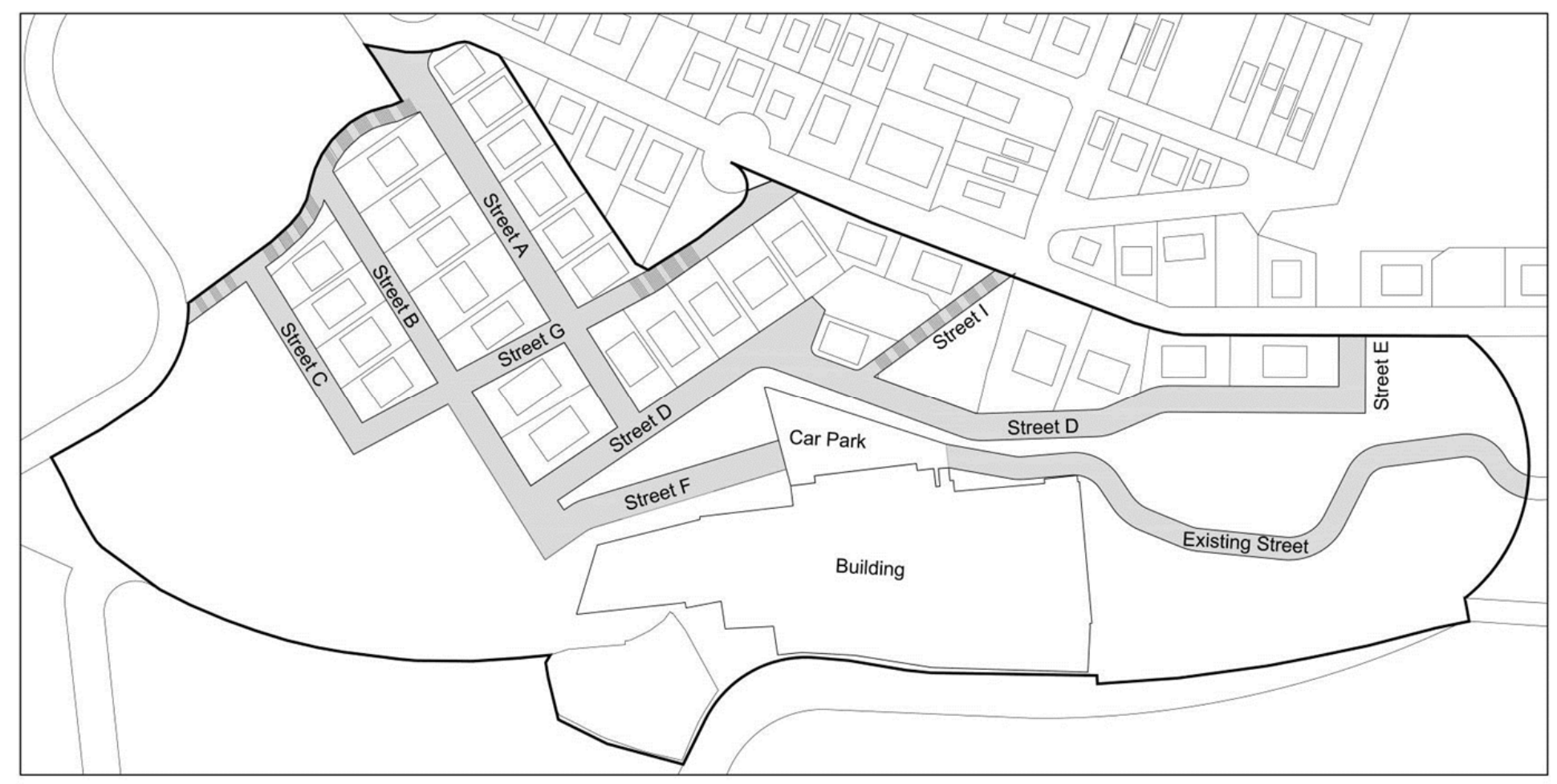

Figure 2. Schematic drawing of case study 2. 


\section{ENVIRONMENTAL ASPECT}

\begin{tabular}{|l|l|l|l|l|l|}
\hline \multicolumn{2}{|l|}{ CASE STUDY 1 } & \multicolumn{4}{l|}{ CASE STUDY 2 } \\
\hline $\mathrm{SV}^{1}$ & $\mathrm{CO}^{2}$ & $\mathrm{SG}^{3}$ & $\mathrm{SV}^{1}$ & $\mathrm{CO}^{2}$ & $\mathrm{SG}^{3}$ \\
\hline
\end{tabular}

\section{ATMOSPHERIC EMISSIONS}

AE-1 Generation of greenhouse gas emissions due to construction machinery and vehicle movements in on-site municipal engineering works.

AE-2 Generation of VOCs and CFCs due to asphalt mixing in on-site municipa engineering activities.

AE-3 Generation of VOCs and CFCs during painting, treatment or finishing in on-site municipal engineering activities

\begin{tabular}{|l|l|l|l|l|l|l|l|}
\hline $1,451.46$ & 3 & 5 & 15 & $9,419.90$ & 5 & 5 & 25 \\
\hline $3,578.00$ & 3 & 5 & 15 & $19,917.09$ & 3 & 5 & 15 \\
\hline 377.72 & 3 & 5 & 15 & $1,123.97$ & 3 & 5 & 15 \\
\hline 92.00 & 3 & 5 & 15 & $6,473.56$ & 5 & 5 & 25 \\
\hline
\end{tabular}

\section{WATER ALTERATION}

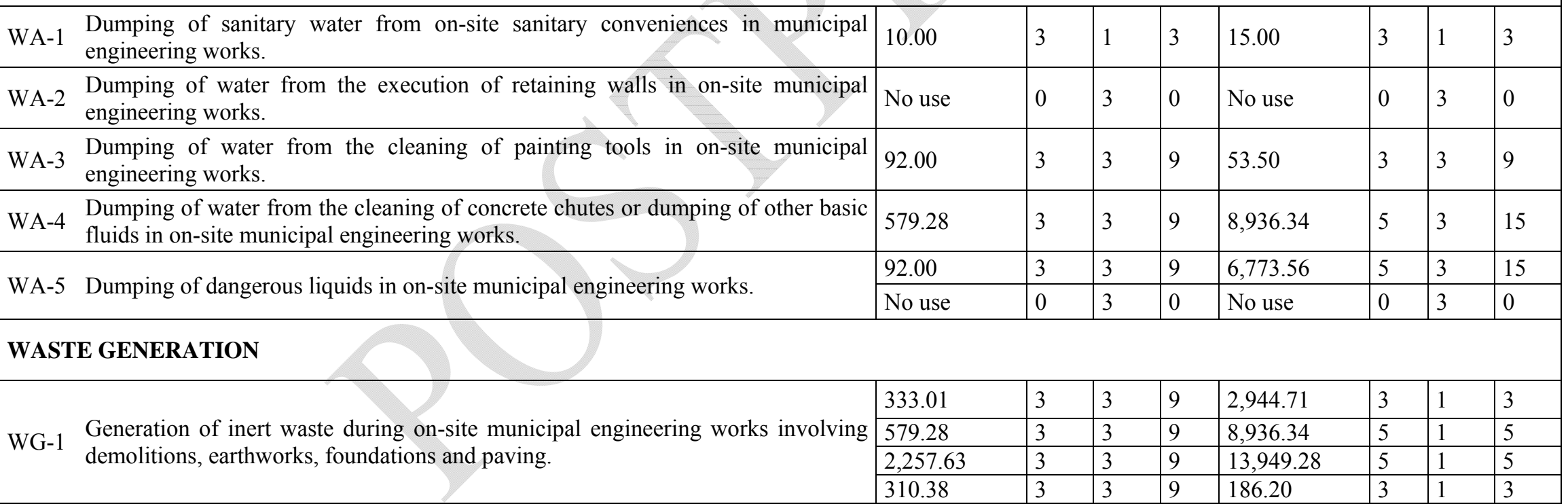




\section{ENVIRONMENTAL ASPECT}

WG-2 Generation of non-special waste from packaging and surplus material in on-site municipal engineering works.

Generation of special waste during on-site municipal engineering works involving

WG-3 non-ecofriendly paints, waterproofing paints, bituminous mixtures and other chemical products.

WG-4 Generation of municipal waste by on-site construction workers in municipa engineering works.

\begin{tabular}{|l|l|l|l|l|l|l|l|}
\hline \multicolumn{2}{|l|}{ CASE STUDY 1 } & \multicolumn{4}{l|}{ CASE STUDY 2 } \\
\hline SV $^{1}$ & $\mathbf{C O}^{\mathbf{2}}$ & $\mathbf{S G}^{3}$ & $\mathbf{S V}^{1}$ & $\mathbf{C O}^{2}$ & $\mathbf{S G}^{3}$ \\
\hline $599,641.71$ & 1 & 3 & 3 & $5,453,030.24$ & 3 & 1 & 3 \\
\hline 469.72 & 3 & 1 & 3 & $7,597.53$ & 5 & 1 & 5 \\
\hline 10.00 & 3 & 3 & 9 & 15.00 & 3 & 1 & 3 \\
\hline
\end{tabular}

\section{SOIL ALTERATION}

SA-1 Land occupancy by provisional on-site facilities in municipal engineering works.

SA-2 Soil alteration from dumping during the use and maintenance of on-site construction machinery and vehicles in municipal engineering works.

SA-3 Soil alteration from dumping during the cleaning of concrete chutes or other basic

SA-3 fluids in on-site municipal engineering works.

SA-4 Soil alteration resulting from dumping during the cleaning of painting tools in onsite municipal engineering works.

SA-5 Soil alteration resulting from dumping during the execution of retaining walls in on-site municipal engineering works.

SA-6 Soil alteration resulting from dumping of dangerous liquids in on-site municipal

SA-6 engineering works.

\section{RESOURCES CONSUMPTION}

RC-1 Water consumption to avoid dust generation in on-site municipal engineering works.

in

RC-2 Electricity consumption in cutting operations during on-site municipal engineering works.

RC-3 Electricity consumption in on-site facilities during municipal engineering works.

\begin{tabular}{|l|l|l|l|l|l|l|l|}
\hline 85.00 & 3 & 5 & 15 & 110.00 & 3 & 1 & 3 \\
\hline $1,451.46$ & 3 & 3 & 9 & $9,419.90$ & 5 & 3 & 15 \\
\hline 579.28 & 3 & 3 & 9 & $8,936.34$ & 5 & 3 & 15 \\
\hline 92.00 & 3 & 3 & 9 & 53.50 & 3 & 3 & 9 \\
\hline No use & 0 & 3 & 0 & No use & 0 & 3 & 0 \\
\hline 92.00 & 3 & 3 & 9 & $6,473.56$ & 5 & 3 & 15 \\
\hline No use & 0 & 3 & 0 & No use & 0 & 3 & 0 \\
\hline
\end{tabular}

No use

\begin{tabular}{|l|l|l|l|l|l|l|l|}
\hline 36.62 & 3 & 1 & 3 & 951.21 & 5 & 1 & 5 \\
\hline 247.67 & 3 & 1 & 3 & $4,134.89$ & 5 & 1 & 5 \\
\hline 10.00 & 3 & 1 & 3 & 15.00 & 3 & 1 & 3 \\
\hline
\end{tabular}




\begin{tabular}{|c|c|c|c|c|c|c|c|c|c|}
\hline \multirow{2}{*}{\multicolumn{2}{|c|}{ ENVIRONMENTAL ASPECT }} & \multicolumn{4}{|c|}{ CASE STUDY 1} & \multicolumn{4}{|c|}{ CASE STUDY 2} \\
\hline & & \multicolumn{2}{|l|}{$\mathbf{S V}^{1}$} & \multirow{2}{*}{\begin{tabular}{|l|}
$\mathbf{C O}^{2}$ \\
5
\end{tabular}} & \multirow{2}{*}{\begin{tabular}{|l|}
$\mathbf{S G}^{3}$ \\
15
\end{tabular}} & \multicolumn{2}{|l|}{$\mathrm{SV}^{1}$} & \multirow{2}{*}{\begin{tabular}{|l|}
$\mathbf{C O}^{2}$ \\
5
\end{tabular}} & \multirow{2}{*}{\begin{tabular}{|l|}
$\mathbf{S G}^{3}$ \\
25
\end{tabular}} \\
\hline $\mathrm{RC}-4$ & Fuel consumption during on-site municipal engineering works. & $1,451.46$ & 3 & & & $9,149.90$ & 5 & & \\
\hline $\mathrm{RC}-5$ & Raw material consumption during on-site municipal engineering works. & $599,641.71$ & 1 & 5 & 5 & $5,453,030.24$ & 3 & 5 & 15 \\
\hline \multicolumn{10}{|c|}{ LOCAL ISSUES } \\
\hline LI-1 & $\begin{array}{l}\text { Generation of noise and vibrations during the execution of channels in on-site } \\
\text { municipal engineering works. }\end{array}$ & 655.60 & 3 & 5 & 15 & $12,605.56$ & 3 & 3 & 9 \\
\hline LI-2 & $\begin{array}{l}\text { Generation of noise and vibrations during demolitions and internal movements of } \\
\text { materials in on-site municipal engineering works. }\end{array}$ & $1,451.46$ & 3 & 5 & 15 & $9,419.90$ & 5 & 3 & 15 \\
\hline & \multirow{2}{*}{$\begin{array}{l}\text { Generation of noise and vibrations during the execution of earth filling and paving } \\
\text { in on-site municipal engineering works. }\end{array}$} & 427.25 & 3 & 5 & 15 & $7,869.19$ & 3 & 3 & 9 \\
\hline & & $2,635.35$ & 3 & 5 & 15 & $10,775.75$ & 3 & 3 & 9 \\
\hline \multirow{2}{*}{ LI-4 } & \multirow{2}{*}{$\begin{array}{l}\text { Generation of smells during painting and paving in on-site municipal engineering } \\
\text { works. }\end{array}$} & 377.72 & 3 & 3 & 9 & $1,123.97$ & 3 & 3 & 9 \\
\hline & & \begin{tabular}{|l|}
92.00 \\
\end{tabular} & 3 & 3 & 9 & 53.50 & 3 & 3 & 9 \\
\hline LI-5 & Generation of smells during gardening in on-site municipal engineering activities. & 72.24 & 3 & 3 & 9 & $2,536.59$ & 5 & 3 & 15 \\
\hline LI-6 & $\begin{array}{l}\text { Dust generation in demolitions and earthworks in on-site municipal engineering } \\
\text { activities. }\end{array}$ & $3,578.00$ & 3 & 5 & 15 & $18,917.09$ & 3 & 5 & 15 \\
\hline LI-7 & $\begin{array}{l}\text { Dust generation during cutting of paving stones in on-site municipal engineering } \\
\text { works. }\end{array}$ & $2,257.63$ & 3 & 5 & 15 & $13,949.28$ & 5 & 5 & 25 \\
\hline LI-8 & $\begin{array}{l}\text { Dust generation during cutting of kerbs and gutters in on-site municipal } \\
\text { engineering works. }\end{array}$ & 310.38 & 3 & 5 & 15 & 186.20 & 3 & 5 & 15 \\
\hline LI-9 & $\begin{array}{l}\text { Dust generation during cutting of asphalt pavements in on-site municipal } \\
\text { engineering works. }\end{array}$ & 23.00 & 1 & 5 & 5 & 97.27 & 3 & 5 & 15 \\
\hline LI-10 & $\begin{array}{l}\text { Dust generation during paving operations (preparation of subgrades, bases and } \\
\text { subbases) in on-site municipal engineering works. }\end{array}$ & 393.98 & 3 & 5 & 15 & $2,069.40$ & 3 & 5 & 15 \\
\hline LI-11 & Landscape alteration by gardening in on-site municipal engineering works. & No existence & 0 & 1 & 0 & Existence & 3 & 1 & 3 \\
\hline
\end{tabular}




\section{ENVIRONMENTAL ASPECT}

\begin{tabular}{|l|l|l|l|l|l|}
\multicolumn{2}{|l|}{ CASE STUDY 1 } & \multicolumn{4}{l|}{ CASE STUDY 2 } \\
\hline $\mathrm{SV}^{1}$ & $\mathrm{CO}^{2}$ & $\mathrm{SG}^{3}$ & $\mathrm{SV}^{1}$ & $\mathrm{CO}^{2}$ & $\mathrm{SG}^{3}$ \\
\hline
\end{tabular}

\section{TRANSPORT ISSUES}

TI-1 Increase in external road traffic due to waste and materials transportation for onsite municipal engineering works.

TI-2 Interference in external road traffic due to the transport of large-size elements for on-site municipal engineering works.

\begin{tabular}{|l|l|l|l|l|l|l|l|}
\hline $599,641.71$ & 1 & 5 & 5 & $5,453,030.24$ & 3 & 1 & 3 \\
\hline No existence & 0 & 3 & 0 & No existence & 0 & 1 & 0 \\
\hline
\end{tabular}

\section{ENVIRONMENTAL ACCIDENTS}

EA-1 Environmental accidents during underground channel execution (drains, sewage systems and services) in on-site municipal engineering works.

EA-2 Environmental accidents during demolitions, earthworks and soil conditioning in on-site municipal engineering works.

EA-3 Environmental accidents caused by fires in machinery in on-site municipa Environmental accide
engineering works.

EA-4 Environmental accidents caused by fires in storage areas in on-site municipa

EA-4 engineering works.

\begin{tabular}{|l|l|l|l|l|l|l|l|}
\hline 655.60 & 3 & 5 & 15 & $12,605.06$ & 3 & 5 & 15 \\
\hline $3,578.00$ & 3 & 5 & 15 & $18,917.09$ & 3 & 5 & 15 \\
\hline $1,451.46$ & 3 & 5 & 15 & $9,419.90$ & 5 & 5 & 25 \\
\hline $599,641.71$ & 1 & 5 & 5 & $5,453,030.24$ & 3 & 5 & 15 \\
\hline
\end{tabular}

\section{EFFECTS ON BIODIVERSITY}

EB-1 Effects on biodiversity due to edaphic soil loss or soil erosion during earthworks in on-site municipal engineering activities.

EB-2 Effects on biodiversity due to vegetation loss in on-site municipal engineering

works.

OVERALL ENVIRONMENTAL IMPACT LEVEL

\begin{tabular}{|c|c|c|c|c|c|c|c|}
\hline $1,720.32$ & 3 & 1 & 3 & $10,930.90$ & 3 & 1 & 3 \\
\hline No existence & 0 & 1 & 0 & Existence & 3 & 1 & 3 \\
\hline No existence & 0 & 1 & 0 & $58,735.00$ & 5 & 1 & 5 \\
\hline
\end{tabular}

${ }^{1} \mathrm{SV}$ : Severity of the environmental impact.

${ }^{2} \mathrm{CO}$ : Concerns of interested parties.

${ }^{3}$ SG: Significance of the environmental impact.

Table 6. Assessment results. 


\section{DISCUSSION OF RESULTS}

According to the assessment results, the overall environmental impact level of case study 1 was 365 , whereas that of the second case study was 459 .

In the first case study and during the pre-construction stage, 15 environmental impacts were highlighted as significant by the model. Although they had moderate severity in this municipal engineering project, environmental impacts related to atmospheric emissions (AE-1 "Generation of greenhouse gas emissions due to construction machinery and vehicle movements", AE-2 "Generation of VOCs and CFCs due to asphalt mixing" and AE-3 "Generation of VOCs and CFCs during painting, treatment or finishing") were deemed significant. This is because the prevention of climate change is a strategic priority for the European Union, so these impacts are of primary concern to all or most interested parties. Thus, corresponding environmental procedures were implemented on-site (i.e. instructions for verifying compulsory inspections and quality labels of construction equipment, machinery and vehicles, instructions for selecting energy-efficient equipment, instructions for encouraging fuel-efficient driving habits, instructions for construction machinery and vehicle maintenance, etc.). As the construction site was located in a densely populated area, an information system for the neighbouring community and a complaint-management system were implemented in order to diminish the significance of environmental impact SA-1 "Land occupancy by provisional on-site facilities". In this case, the environmental impact had a moderate severity. However, the concerns of internal interested parties were considered to be primary, as the municipal engineering site affects pedestrian thoroughfares. The assessment of this municipal engineering project also found that environmental impact RC-4 "Fuel consumption" was significant. Therefore, preventive actions (similar to those devised to diminish carbon dioxide emissions) were planned and subsequently implemented on-site. This environmental impact had moderate severity, but in accordance with the priorities stated in current policies on natural resource conservation, concerns of interested parties were deemed to be important. Three environmental impacts related to generation of noise and vibrations were found to be significant (LI-1 "Generation of noise and vibrations during the execution of channels", LI-2 "Generation of noise and vibrations during demolitions and internal movements of materials" and LI-3 "Generation of noise and vibrations during the execution of earth filling and pavements"). Besides having moderate severity, these environmental impacts were deemed significant because the municipal engineering work is located in a high acoustic comfort area (near a hospital). In this case, noise mitigation measures were implemented on-site (i.e. instructions for the maintenance of construction equipment and tools so they can run at normal manufacturer's operating specifications, instructions for installing temporary barriers, instructions for coordinating the on-site work schedule, etc.). In the assessment of the first case study, four significant environmental impacts were identified in relation to dust generation (LI-6 "Dust generation in demolition and earthwork", LI-7 "Dust generation during cutting of paving stones", LI-8 "Dust generation during cutting of kerbs and gutters" and LI-10 "Dust generation during paving - preparation of subgrades, bases and subbases"). These environmental impacts are significant because of their moderate severity and the fact that they represent a primary concern to all or most interested parties. Besides causing health risks to surrounding people, internal interested parties are also concerned because dust 
generation may require increased cleaning. In this case, dust mitigation measures (i.e. instructions for wetting vehicle and machinery paths, instructions for handling and storing dusty materials, etc.) were taken into account. Environmental impact RC-4 "Fuel consumption" was also found to have a significant impact. Therefore, preventive actions (similar to those devised to diminish carbon dioxide emissions) were planned and then implemented on-site. This environmental impact had moderate severity, but in accordance with the priorities stated in current policies on natural resource conservation, the concerns of interested parties were deemed to be important. As environmental impacts related to accidents may have greater consequences in densely populated areas, the model highlighted the significance of environmental impacts EA-1 "Environmental accidents during underground channel execution", EA-2 "Environmental accidents during demolitions, earthworks and soil conditioning" and EA-3 "Environmental accidents caused by fires in machinery". For these cases, environmental procedures designed for use in potential emergency situations were implemented (i.e. instructions in case of electrical accidents, instructions in case of explosions and fires, etc.).

In the second case study and during the pre-construction stage, 21 environmental impacts were highlighted as significant by the model. The three environmental impacts related to the atmospheric emissions category (AE-1 "Generation of greenhouse gas emissions due to construction machinery and vehicle movements", AE-2 "Generation of VOCs and CFCs due to asphalt mixing" and AE-3 "Generation of VOCs and CFCs during painting, treatment or finishing") were found to be significant. Consequently, corresponding work instructions (similar to those planned in this area for case study 1) were implemented on-site. The assessment of the second case study also led to the identification of two significant environmental impacts related to water alteration (WA4 "Dumping of water from cleaning concrete chutes or dumping of other basic fluids" and WA-5 "Dumping of dangerous liquids") and three significant environmental impacts related to soil alteration (SA-2 "Soil alteration resulting from dumping during the use and maintenance of on-site construction machinery and vehicles", SA-3 "Soil alteration resulting from dumping during cleaning of concrete chutes or other basic fluids" and SA-6 "Soil alteration resulting from dumping of dangerous liquids"). Although of secondary concern to all or most interested parties as the plan was to use the existing sewage system during on-site construction works, all these environmental impacts were found to have major severity. In this case, procedures related to on-site wastewater management were implemented (i.e. instructions for cleaning concrete chutes, instructions for the on-site maintenance of construction machinery and vehicles, instructions for accidental dumping of dangerous liquids, etc.). As in case study 1, environmental impact RC-4 "Fuel consumption" was found to have an extremely significant impact. However, the severity of this environmental impact was found to be higher than in case study 1 . In this case, the environmental impact RC-5 "Raw material consumption" was significant, mainly because the recycled content of raw materials was not specified in the construction project documents. Instructions related to raw material and fuel saving were implemented on site (i.e. instructions for materials purchasing, instructions for materials storage, instructions for selecting energy-efficient equipment, instructions for encouraging fuel-efficient driving habits, etc.). As in the first case study, environmental impact LI-2 "Generation of noise and vibrations during demolitions and internal movements of materials" was found to be significant due to its severity, and corresponding preventive actions were implemented. One environmental impact related 
to the generation of smells and five environmental impacts related to dust generation were found to be significant in the second case study (LI-5 "Generation of smells during gardening works", LI-6 "Dust generation in demolitions and earthworks", LI-7 "Dust generation during cutting of paving stones", LI-8 "Dust generation during cutting of kerbs and gutters", LI-9 "Dust generation during cutting of asphalt pavements" and LI10 "Dust generation during paving operations - preparation of subgrades, bases and subbases"). With moderate or major severity, these environmental impacts were deemed to be significant, because the site is located in a residential area. Thus, on-site instructions similar to those planned in the first case study were implemented on-site. Unlike in case study 1, specific on-site procedures were also implemented to minimize the significance of environmental impact LI-5 and LI-10 (i.e. instructions for paving with asphalt, instructions for stockpiles, instructions for handling dusty materials, etc.). Finally, the model highlighted the significance of the four environmental impacts related to the environmental accidents category (EA-1 "Environmental accidents during underground channel execution: drains, sewage system and services", EA-2 "Environmental accidents during demolitions, earthworks and soil conditioning", EA-3 "Environmental accidents caused by fires in machinery" and EA-4 "Environmental accidents caused by fires in storage areas") and corresponding on-site work instructions (similar to those planned in case study 1) were implemented. These environmental impacts were significant due to their moderate or major severity and the fact that they are a primary concern to all or most interested parties because the site is located in an urban area with nearby occupied buildings.

\section{CONCLUSIONS}

This research provides a model for dealing with potentially adverse on-site environmental impacts during the pre-construction stage of municipal engineering works. The model is useful within the framework of EIAs of municipal engineering projects, as it facilitates the early identification and evaluation of on-site environmental impacts. The model is also valuable for construction companies that work in the field of municipal engineering projects and are willing to implement an environmental management system. It can also simply help construction companies to improve their on-site environmental performance in municipal engineering projects by prioritizing which environmental impacts should be addressed on-site. As the model ranks and highlights significant environmental impacts of on-site municipal engineering works in advance, preventive actions can be planned and implemented to mitigate adverse effects. The model also allows a comparison of the overall environmental impact level and the absolute importance of a particular environmental aspect in various municipal engineering projects or alternatives.

This paper extends the approach presented in Gangolells et al. $(2009,2011)$ from residential building units to municipal engineering projects. This research first identified 42 significant on-site environmental impacts of municipal engineering works using overall significance rating matrixes based on an assessment of impact duration, impact scale and probability of occurrence. Then, to assess the concerns of the interested parties and the severity of each of the environmental aspects in the model, 46 indicators were developed using the content analysis method. To ensure the objectivity of the 
assessment process, both direct and indirect indicators were always based on data from the project documents. Finally, significance limits were determined on the basis of a statistical analysis of 25 new-build and remodelling municipal engineering projects. Another key contribution of this research to the body of knowledge is the characterization, in quantitative terms, of municipal engineering projects. The establishment of a benchmark against which to judge other projects also configures an important contribution.

During the identification and assessment process of on-site environmental aspects related to municipal engineering projects, guidance stated in ISO 14004:2004 was used. Further research is needed in order to include other attributes such as synergy, reversibility, accumulation and intensity as this would significantly improve the model. The model is developed from an European perspective and thus it may not accurately reflect the construction practices that are widespread in other countries. However, the methodology can be easily replicated to other contexts. As a first step, the methodology proposes an exhaustive preliminary analysis with a process-oriented approach. By including other construction techniques and systems in the process-oriented approach, on-site environmental aspects of municipal engineering projects can be tailored to regional specificities. Developed indicators and significance limits can also be adapted to other countries by following the suggested approach, assuming as a baseline the typical or average performance levels in typical construction projects executed within the area of applicability. Taking into account that assessing the on-site environmental impacts of a municipal engineering project may involve a great amount of time, further research is needed to speed up the assessment process. The time devoted to the assessment of each municipal engineering project could be substantially reduced by implementing the methodology in a web-based information and knowledge management system with databases reusing indicator calculations. Time and effort could be saved even more by automatically importing all the data needed from the tools used during the design process (i.e. all the data related to the bill of quantities can be automatically imported from the Building Information Models). Once optimized, the model could be used in any municipal engineering project regardless of its size or complexity. Further research also needs to be carried out to link on-site environmental impacts and health and safety risks, as the notion of injury equally applies to damage to the life and health of employees, the surrounding population and the environment (Gangolells et al., 2013). In this way, the implementation of integrated environmental and health and safety management systems in construction companies could be enhanced by simultaneously identifying, assessing and operationally controlling environmental aspects and health and safety risks in municipal engineering projects (Gangolells et al., 2012; 2013). In order to achieve a more accurate, realistic, and consistent assessment, further research should also include the analysis of cumulative impacts. In this sense, the development and application of geographic information systems (GIS) and remote sensing (RS) databases could be useful. Finally and taking into account the contribution of the construction sector to sustainable development and sustainable communities, further research should also examine the socio-economic impacts related to municipal engineering projects and investigate the possibility of including them within the developed methodology. 


\section{REFERENCES}

Androulidakis I, Karakassis I. Evaluation of the EIA system performance in Greece, using quality indicators. Environmental Impact Assessment Review 2006; 26(3): 242-56.

Badr EA, Zahran AA, Cashmore M. Benchmarking performance: environmental impact statements in Egypt. Environmental Impact Assessment Review 2011; 31(3): 279-85.

Canelas L, Almansa P, Merchan M, Cifuentes P. Quality of environmental impact statements in Portugal and Spain. Environmental Impact Assessment Review 2005; 25(3): 217-25.

Gangolells M, Casals M. An ontology-based approach for on-site integrated environmental and health and safety management. Revista Ingeniería de Construcción 2012; 27(3): 103-27.

Gangolells M, Casals M, Forcada N, Fuertes A, Roca X. A model for enhancing integrated identification, assessment and operational control of on-site environmental impacts and health and safety risks in construction firms. Journal of Construction Engineering and Management 2013; 139(2): 138-47.

Gangolells M, Casals M, Gassó S, Forcada N, Roca X, Fuertes A. A methodology for predicting the severity of environmental impacts related to the construction process of residential buildings. Building and Environment 2009; 44(3): 558-71.

Gangolells M, Casals M, Gassó S, Forcada N, Roca X, Fuertes A. Assessing concerns of interested parties when predicting the significance of environmental impacts related to the construction process of residential buildings. Building and Environment 2011; 46(5): 1023-37.

Gómez-Navarro T, García-Melón $M$, Acuña-Dutra S, Díaz-Martín D. An environmental pressure index proposal for urban development planning based on the analytic network process. Environmental Impact Assessment Review 2009; 29(5): 319_ 29.

Fuertes A, Casals M, Gangolells M, Forcada N, Macarulla M, Roca X. An Environmental Impact Causal Model for improving the environmental performance of construction processes. Journal of Cleaner Production 2013; 52: 425-437.

European Commission. Interpretation of definitions of certain project categories of annex I and II of the EIA Directive. European Commission 2008. $<\mathrm{http} / /$ ec.europa.eu/environment/eia/pdf/interpretation_eia.pdf $>$ (Sep 7, 2011).

European Union. Regulation (EC) No 1221/2009 of the European Parliament and of the Council of 25 November 2009 on the voluntary participation by organisations in a Community eco-management and audit scheme (EMAS), repealing Regulation (EC) No 761/2001 and Commission Decisions 2001/681/EC and 2006/193/EC.

European Union. Directive 2011/92/EU of the European Parliament and of the Council of 13 December 2011 on the assessment of the effects of certain public and private projects on the environment.

He X, Liu Y, Li T, Chen J. Does the Rapid Development of China's Urban Residential Buildings Matter for the Environment? Building and Environment 2013; 64: 130-137. Holsti OR. Content analysis for the social sciences and humanities. Reading MA: Addison-Wesley; 1969.

International Standard Organisation. ISO 14004:2004 Environmental management systems: General guidelines on principles, systems and support techniques. 
Jay S, Jones C, Slinn P, Wood C. Environmental impact assessment: Retrospect and prospect. Environmental Impact Assessment Review 2007; 27(4): 287-300.

Kruopienė J, Židonienė S, Dvarionienė J. Current practice and shortcomings of EIA in Lithuania. Environmental Impact Assessment Review 2009; 29(5): 305-9.

Lundberg K, Balfors B, Folkeson L. Identification of environmental aspects in an EMS context: a methodological framework for the Swedish National Rail Administration. Journal of Cleaner Production 2007; 15(5): 385-94.

Martínez Orozco JM. El sistema español de evaluación de impacto ambiental; nuestra posición en el mundo. Informes de la Construcción 2006; 58(504): 29-38.

Nadeem O, Hameed R. Evaluation of environmental impact assessment system in Pakistan. Environmental Impact Assessment Review 2008; 28(8): 562-71.

Peterson K. Quality of environmental impact statements and variability of scrutiny by reviewers. Environmental Impact Assessment Review 2010; 30(3): 169-76.

Rattleff $\mathrm{P}$. The reliability of content analysis of computer conference communication. Computers \& Education 2007; 49(2): 230-42.

Riera MG, Rey E. A multi-criteria approach to compare urban renewal scenarios for an existing neighborhood. Case study in Lausanne (Switzerland). Building and Environment 2013; 65: 58-70.

Shen ML, Wu Y, Zhang X. Key Assessment Indicators for the Sustainability of Infrastructure Projects. Journal of Construction Engineering and Management 2011; 137(6): 441-51.

Tennøy A, Kværner J, Gjerstad K. Uncertainty in environmental impact assessment predictions: the need for better communication and more transparency. Impact Assessment and Project Appraisal 2006; 24(1): 45-56.

Toro J, Requena I, Zamorano M. Environmental impact assessment in Colombia: Critical analysis and proposals for improvement. Environmental Impact Assessment Review 2010; 30(4): 247-61.

Toro J, Requena I, Zamorano M. Determining vulnerability importance in environmental impact assessment. The case of Colombia. Environmental Impact Assessment Review 2012; 32 (1): 107-17.

United Nations Human Settlements Programme. Global report on human settlements 2009. Planning sustainable cities. London: Un-Habitat and Earthscan 2009. $<$ http://www.unhabitat.org/downloads/docs/GRHS2009/GRHS.2009.pdf >. (Sep 7, 2011).

Zobel T, Burman JO. Factors of importance in identification and assessment of environmental aspects in an EMS context: Experiences in Swedish organizations. Journal of Cleaner Production 2004; 12(1): 13-27. 